\title{
On a sparse pressure-flow rate condensation of rigid circulation models
}

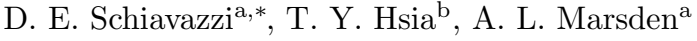 \\ ${ }^{a}$ Mechanical and Aerospace Engineering Department, University of California, San Diego, USA \\ ${ }^{b}$ Great Ormond Street Hospital for Children and UCL Institute of Cardiovascular Science, London, UK
}

\begin{abstract}
Cardiovascular simulation has shown potential value in clinical decision-making, providing a framework to assess changes in hemodynamics produced by physiological and surgical alterations. State-of-the-art predictions are provided by deterministic multiscale numerical approaches coupling 3D finite element Navier Stokes simulations to lumped parameter circulation models governed by ODEs. Development of next-generation stochastic multiscale models whose parameters can be learned from available clinical data under uncertainty constitutes a research challenge made more difficult by the high computational cost typically associated with the solution of these models. We present a methodology for constructing reduced representations that condense the behavior of 3D anatomical models using outlet pressure-flow polynomial surrogates, based on multiscale model solutions spanning several heart cycles. Relevance vector machine regression is compared with maximum likelihood estimation, showing that sparse pressure/flow rate approximations offer superior performance in producing working surrogate models to be included in lumped circulation networks. Sensitivities of outlets flow rates are also quantified through a Sobol' decomposition of their total variance encoded in the orthogonal polynomial expansion. Finally, we show that augmented lumped parameter models including the proposed surrogates accurately reproduce the response of multiscale models they were derived from. In particular, results are presented for models of the coronary circulation with closed loop boundary conditions and the abdominal aorta with open loop boundary conditions.
\end{abstract}

Keywords: Cardiovascular simulation, sparse regression, reduced order models, relevance vector machines, multiscale modeling of cardiovascular systems.

\section{Introduction}

Three-dimensional simulations of vascular hemodynamics are widely used, with increasing clinical implication, in applications ranging from pediatric cardiology to coronary artery and aneurysm hemodynamics. Before these models can be used to complement clinical decision making, vascular geometry, material properties and boundary conditions need to be estimated from clinical data. Unfortunately, the significant computational cost of simulations severely limits the use of parameter estimation, uncertainty quantification and optimization procedures, all requiring repeated model evaluations needed to produce reasonable patient-specific estimates of model parameters. We argue that such characterization of model parameters should also be determined stochastically, producing associated confidence metrics on simulation results. This typically leads to a further increase in the number of simulations needed, for example, in Bayesian estimation approaches.

Multiscale cardiovascular models (see, e.g., Formaggia et al., 2001, Moghadam et al., 2013), for example, couple three-dimensional discrete finite element simulations with 1D or lumped parameter descriptions of the patient circulation. Lumped parameter models, obtained by linearizing the Navier-Stokes equations around

*Corresponding author

Preprint submitted to Elsevier

November 9, 2015

(C) 2015. This manuscript version is made available under the Elsevier user license http://www.elsevier.com/open-access/userlicense/1.0/ 
rest conditions (Milišić and Quarteroni, 2004), are formulated through systems of ODEs analogous to those describing the evolution of current and voltage in an electrical circuit and belong to two main families, i.e., open or closed loop boundary conditions. Open loop boundary conditions (see, e.g., Vignon-Clementel et al., 2006) are circuit models, such as RCR, that are applied at each outlet face and end at ground, while closed loop boundary conditions (see, e.g., Corsini et al., 2014) connect back to the model inlet to mimic the entire circulation. We will refer to the latter models as circulation or LPN models in what follows. Similar systems can also be assembled by coupling 3D CFD models with 1D peripheral counterparts. While techniques for parameter estimation and data assimilation in lumped circulation networks have been proposed in the literature (see, e.g., Spilker and Taylor, 2010, Revie et al., 2013, Sughimoto et al., 2013, Yu et al., 2001, Xiao et al., 2002, Deswysen, 1977, Clark et al., 1980, Deswysen et al., 1980, McInnis et al., 1985, Shimooka et al., 1991, Avanzolini et al., 1992, Ruchti et al., 1993, Yu et al., 1998), the computational cost of solving the coupled 0D/3D problem has prevented extension of these techniques to multiscale models.

A viable approach to overcome this difficulty is to construct a reduced order model that can replace a full finite element solution at a fraction of the computational cost. A significant number of contributions on this topic can be found in the literature and the list that follows is by no means comprehensive.

A review of reduced order modeling approaches is presented in Benner et al. (2013) addressing and comparing model reduction techniques for non-parameterized and parameterized systems developed in the proper orthogonal decomposition (POD) and the control theory communities, respectively. It discusses computing the trial and test spanning sets for Petrov-Galerkin projection basis using three approaches, rational interpolation, balanced truncation and POD. Strategies for sampling in parameter space to minimize errors between the original high-fidelity model and its reduced order representation are also critically reviewed.

An extensive treatment of reduced basis approximations for affinely parameterized elliptic coercive partial differential equations is presented in Rozza et al. (2008), where the authors discuss an online-offline projection of a full finite element solution onto the space generated by solution snapshots identified through greedy sampling strategies. A review of recent developments in this direction is also presented in Chen et al. (2015).

In the context of interpolatory approximation of the response functions for general SISO (single-input, single-output) and MIMO (multiple-input, multiple-output) systems, an extensive review can be found in Antoulas et al. (2010), also addressing situations where the Loewner data-driven framework is used for highfidelity systems characterized through a reduced number of inputs and outputs. A recent contribution, in this context, discusses the development of rational macro-models for one-dimensional blood flow in arteries (Ferranti et al., 2015).

Balanced truncation approaches are reviewed in Gugercin and Antoulas (2004), while a vast literature is available on POD methods, starting from the first applications to turbulent flow in the late 60's to more recent developments (see, e.g., Berkooz et al., 1993, Rowley, 2005).

Finally, additional recent work on reduced order modeling with cardiovascular applications can be found in McLeod et al. (2010), Guibert et al. (2014), Colciago (2014), Ballarin (2015).

Our approach separates the finite element model and its boundary circulation network in two systems with coupling occurring at the interface, where pressure/flow rate information is (implicitly or explicitly) exchanged at every solution time step. In particular, the LPN model receives outlet flow rates from the finite element model and provides pressures back to the same outlets for the next time step. Conversely, the 3D model receives outlet pressures from the LPN and provides updated local distributions of pressure and velocities, resulting in new outlet flow rates. This way of representing a multiscale model, i.e., consisting of two subsystems continuously exchanging pressure/flow rate information, inspires model reduction approaches that preserve the exchange of information at the interface. We therefore propose a methodology for building outlet pressure-flow rate approximants of discrete models. We demonstrate that use of sparse regressors is paramount to building condensed representations that can be successfully integrated into ODE solvers for boundary circulation models.

The problem of constructing a pressure/flow rate surrogate of a discrete anatomical model is presented in Section 2, formulated as an orthogonal multivariate polynomial regression problem. We also discuss the main assumptions behind this approach, mainly related to treatment of inertia and compliance phenomena. 
Section 3 presents two methodologies for estimating the expansion coefficients, namely maximum likelihood estimation through Ordinary Least Squares (OLS see, e.g., Banks et al., 2014) and Relevance Vector Machine regression (RVM) (Tipping, 2001, Tipping et al., 2003). Pressure-flow surrogates are constructed in Section 4, while Section 5 shows how outlet flow rate sensitivities and amplification of pressure perturbations though the approximant are affected by constraining the sparsity of the resultant representation. Finally, conclusions are presented in Section 6.

\section{Problem statement}

Consider a region $\Omega \subset \mathbb{R}^{3}$ occupied by a three-dimensional representation of the vasculature. The boundary of $\Omega$ is denoted by $\partial \Omega$ and partitioned into the following disjoint sets: walls $\Gamma^{w}=\left\{\Gamma_{j}^{w}, j=\right.$ $\left.1, \ldots, n_{w}\right\}$, inlets $\Gamma^{i}=\left\{\Gamma_{j}^{i}, j=1, \ldots, n_{i}\right\}$ and outlets $\boldsymbol{\Gamma}^{o}=\left\{\Gamma_{i}^{o}, i=1, \ldots, n_{o}\right\}$. In what follows, the set $\boldsymbol{\Gamma}=\left\{\Gamma_{j}, j=1, \ldots, n\right\}$ will be used to generically refer both to model inlets or outlets or, in other words, $\boldsymbol{\Gamma}=\boldsymbol{\Gamma}^{i} \cup \boldsymbol{\Gamma}^{o}$ and $n=n_{i}+n_{o}$. Average pressures and flow rates at the generic $\Gamma_{i}$ are characterized using the random vectors $\mathbf{p}=\left(p_{1}, \ldots, p_{n}\right) \in \boldsymbol{\Lambda}_{\boldsymbol{p}} \subset \mathbb{R}^{n}$ and $\mathbf{q}=\left(q_{1}, \ldots, q_{n}\right) \in \boldsymbol{\Lambda}_{\boldsymbol{q}} \subset \mathbb{R}^{n}$ with joint distribution $\rho(\mathbf{p})$ and $\rho(\mathbf{q})$, respectively, defined over the probability triplet $(\Xi, \mathcal{F}, \mathcal{P})$. Here, $\Xi$ is the sample space, $\mathcal{F}$ is a $\sigma$-algebra of possible events, and $\mathcal{P}$ denotes a probability measure on $\mathcal{F}$. The generic average outlet pressure $p_{i}$ and flow rate $q_{i}$ are also defined as follows:

$$
p_{i}=\frac{1}{\left|\Gamma_{i}\right|} \int_{\Gamma_{i}} p d \Gamma_{i}, \quad q_{i}=\int_{\Gamma_{i}}\left(\mathbf{v} \cdot \mathbf{n}_{i}\right) d \Gamma_{i}
$$

where $p, \mathbf{v}$ are the 3D model pressure and velocity field, respectively, while $\mathbf{n}_{i}$ is the normal to the interface $\Gamma_{i}$, assumed planar. We assume that the discrete 3D model can be replaced by a relationship between pressures and flow rates of the form:

$$
q_{i}(t)=f(\mathbf{p}, t, \gamma)=\sum_{j=1}^{n_{b}} \alpha_{j, i}(t, \gamma) \phi_{j}(\mathbf{p}, t, \gamma), i=1, \ldots, n
$$

where $t$ represents time, $\boldsymbol{\alpha}_{i}=\left(\alpha_{1, i}, \ldots, \alpha_{n_{b}, i}\right) \in \mathbb{R}^{n_{b}}$ is a vector of expansion coefficients characterizing the flow rate at the generic $\Gamma_{i}, \phi_{j}$ is a set of basis functions (yet to be chosen, see next paragraph) with cardinality $n_{b}$, assuming values over $\mathbf{p}, t$ and $\gamma$, a vector of additional variables. A possible example of additional variables in $\gamma$ is the total model volume (initial model volume plus net flow rate through the model integrated over time) in deformable wall simulations. At this point, we assume independence from $t$ and $\gamma$ in (2). The first assumption, time independency, holds in situations characterized by limited inertia effects. We refer the reader to Section 2.1 for a discussion on the contribution of inertance to the pressure drop for two extreme vessel geometries and physiological flow conditions. The second assumption is instead related to the choice of rigid wall models. Although the approach presented in the next sections can be extended to deformable wall models by properly introducing additional variables, we leave the demonstration of this to future studies. The expansion in (2) can therefore be simplified to:

$$
q_{i}=\sum_{j=1}^{n_{b}} \alpha_{j, i} \phi_{j}(\mathbf{p}), i=1, \ldots, n .
$$

Multivariate Legendre polynomials, combined with a linear transformation to $[-1,1]$ are used, in this work, as the basis functions $\phi_{j}, j=1, \ldots, p$. These polynomials are orthogonal with respect to a uniform weight function $w\left(x_{i}\right)=0.5, x_{i} \in[-1,1] \subset \mathbb{R}$, and satisfy the three-term recurrence formula:

$$
(j+1) \bar{L}_{j+1}\left(x_{i}\right)=(2 j+1) x_{i} \bar{L}_{j}\left(x_{i}\right)-j \bar{L}_{j-1}\left(x_{i}\right)
$$


Denoting $L_{j}\left(x_{i}\right)=\bar{L}_{j}\left(x_{i}\right) / \sqrt{2 /(2 j+1)}, L_{k}\left(x_{i}\right)=\bar{L}_{k}\left(x_{i}\right) / \sqrt{2 /(2 k+1)}$, i.e., the rescaled Legendre polynomials of order $j$ and $k$, respectively, their orthonormality can be stated as:

$$
\left\langle L_{j}, L_{k}\right\rangle_{\mathcal{L}}=\int_{[-1,1]} L_{j} L_{k} d \mathbf{x}=\delta_{j, k}
$$

where $\langle\cdot\rangle_{\mathcal{L}}$ denotes the inner product in the appropriate Hilbert space $\mathcal{L}$ and $\delta_{i, j}$ the Kronecker symbol. Finally, we express the generic basis function $\phi_{j}$ as a tensor product of Legendre polynomials over normalized pressures as follows:

$$
\phi_{j}\left(p_{1}, \ldots, p_{n}\right)=L_{j_{1}}\left[T_{1}\left(p_{1}\right)\right] L_{j_{2}}\left[T_{2}\left(p_{2}\right)\right] \ldots L_{j_{n}}\left[T_{n}\left(p_{n}\right)\right]=\mathbf{L}_{\mathbf{j}}(\mathbf{x}) \in \bigotimes_{k=1}^{n} \mathcal{L}_{k}
$$

where the index $j$ establishes an ordering (e.g., lexicographic with repeated indexes) of the multi-index $\mathbf{j}=\left(j_{1}, j_{2}, \ldots, j_{n}\right)$, containing the degrees of the Legendre polynomials in each dimension. In this study, we conserve the total polynomial order of approximation in $n$ dimensions, i.e., the cardinality of the basis set for the order $r$ is equal to $n_{b}=(n+r) ! / n ! r$ !. In practical applications, as will be discussed in Section 4 , $r=1$ is sufficient. In contrast, for $r$ of the same order of $n$, the fast growth in $n_{b}$ would prevent any practical use of this methodology for a large number of outlets. The transformation $T_{i}: \mathbb{R} \rightarrow[-1,1]$ is selected to preserve the origin (i.e., $p_{i}=0$ ), resulting in zero flow rate for zero outlet pressures, for cases where $\alpha_{1}=0$.

The reason behind our choice of Legendre polynomials will become apparent in the following sections and relates to the ease of decomposing the variance of $q_{i}, i=1, \ldots, n$ at each outlet after computing the expansion (3). Moreover, the coefficients $\alpha_{j, i}, j=1, \ldots, n_{b}$ encode the flow rate statistics at the generic $\Gamma_{i}$ :

$$
\begin{aligned}
& \mu_{i}=\frac{1}{\left|\Lambda_{\mathbf{p}}\right|} \int_{\Lambda_{\mathbf{p}}} q_{i} d \Lambda_{p} \approx \int_{[-1,1]^{n}}\left(\sum_{j=1}^{n_{b}} \alpha_{j, i} \mathbf{L}_{j}(\mathbf{x})\right) d \mathbf{x}=\alpha_{1, i}, \\
& \sigma_{i}^{2}=\frac{1}{\left|\Lambda_{\mathbf{p}}\right|} \int_{\Lambda_{\mathbf{p}}}\left(q_{i}-\mu_{i}\right)^{2} d \Lambda_{\mathbf{p}} \approx \int_{[-1,1]^{n}}\left[\sum_{j=1}^{n_{b}} \alpha_{j, i} \mathbf{L}_{j}(\mathbf{x})\right]^{2} d \mathbf{x}-\mu_{i}^{2}=\sum_{j=2}^{n_{b}} \alpha_{j, i}^{2},
\end{aligned}
$$

where $\left|\Lambda_{\mathbf{p}}\right|$ denotes the volume of $\Lambda_{\mathbf{p}}$.

We assume that a solution of the multiscale model is available for several heart cycles until a periodic response is obtained. We also define the matrices $\mathbf{P}$ and $\mathbf{Q}$ such that, at every converged timestep, a row with the pressures at $\Gamma_{i}$ is appended to $\mathbf{P}$, while flow rates are appended to $\mathbf{Q}$, respectively. This way, $Q_{i, j}$ contains the flow rate computed at outlet $j$ for time step $i$, while $P_{i, j}$ contains the associated averaged pressure. For a total of $m_{t}$ time steps, we therefore have $\mathbf{P}, \mathbf{Q} \in \mathbb{R}^{m_{t} \times n}$.

Before proceeding further, we discard the initial $m_{w}$ time steps, that represent the initial transient conditions. This step is important to avoid corruption of the approximation with values of flow rate/pressure that depend on the initial conditions. The number of samples $m$ used to determine the expansion (3) is then randomly sub-sampled from the remaining $m_{t}-m_{w}$ pressure/flow rows and the approximation problem finally cast in matrix form:

$$
\mathbf{q}_{i}=\mathbf{\Phi} \boldsymbol{\alpha}_{i}
$$

where the selected flow rates at $\Gamma_{i}$ are $\mathbf{q}_{i}=\left(Q_{s_{1}, i}, Q_{s_{2}, i}, \ldots, Q_{s_{m}, i}\right)$ and $\left(s_{1}, s_{2}, \ldots, s_{m}\right)$ is a random subsample of $\left(1,2, \ldots, m_{t}-m_{w}\right), \boldsymbol{\alpha}_{i}=\left(\alpha_{1, i}, \ldots, \alpha_{n_{b}, i}\right)$ is a vector of expansion coefficients for $\Gamma_{i}$ and the matrix $\boldsymbol{\Phi}$ is expressed as:

$$
\Phi_{k, l}=\prod_{\substack{j_{s} \in \mathbf{l}, i=1, \ldots, n}} L_{j_{s}}\left[T_{s_{k}}\left(P_{s_{k}, i}\right)\right]
$$




\subsection{Contribution of inertance}

In this section, we provide quantitative arguments to justify our choice to disregard the dependence between outlet pressures and time derivative of flow rate. Consider a schematic representation of an idealized cylinder filled with fluid in motion as an assembly of a resistance and inductance (or inertance) element, as shown in Figure 2. The pressure drop between inlet and outlet in a straight tube with circular cross section subject to laminar flow conditions is equal to:

$$
\left(p_{2}-p_{1}\right)=\Delta p=I \dot{q}+R q=\left(\frac{\rho L}{\pi r^{2}}\right) \dot{q}+\left(\frac{8 \nu L}{\pi r^{4}}\right) q .
$$

We shall now consider two different scenarios representing idealized circulation in the aorta and coronary arteries of an adult patient, respectively. For each scenario, we selected an appropriate flow rate curve and estimate the contribution to the overall pressure drop by the resistance and inertance elements. Figure 3 shows that, for the scenarios summarized in Table 1, the total pressure drop is dominated by the contribution from resistance. This fact supports our choice to construct surrogates from outlet flow rates, neglecting terms related to their time derivative.

\section{Estimation of Legendre expansions}

The expansion coefficient vector $\boldsymbol{\alpha}_{i}$ at $\Gamma_{i}$ can be determined using several approaches depending on the values of $n, m, n_{b}$ and additional information on its expected structure.

Two alternative methodologies are discussed here, characterized by different underlying assumptions. The first approach consists of computing a maximum likelihood estimator (MLE) for $\boldsymbol{\alpha}_{i}$. For a linear statistical model with independent noise components of uniform variance, this is equivalent (see, e.g., Banks et al., 2014) to minimizing the squared approximation error by ordinary least squares (OLS). In this approach, no assumption is made related to the expected structure of the components $\alpha_{j, i}$, thus allowing for possible interactions between all the outlets. In other words, using this approach we implicitly assume that the pressure at every outlet may affect flow rate at an individual outlet $\Gamma_{i}$.

The second approach uses Relevance Vector Machines (RVM) (Tipping, 2001, Tipping et al., 2003), which promotes small expansion coefficients through specification of properly selected hyperpriors. This choice is motivated by the physical intuition that the flow rate at $\Gamma_{i}$ depends on the pressure distribution at only a limited number of other outlets.

Details of these two approaches are discussed in the following Sections. In particular, we discuss how first and second order statistics for these estimators are derived.

\subsection{OLS estimator}

Computation of the expansion coefficients $\boldsymbol{\alpha}_{i}$ for the generic $\Gamma_{i}$ is performed by minimization of the squared error:

$$
e_{i}=\sum_{j=1}^{m}\left[\mathbf{q}_{i}-\boldsymbol{\Phi} \boldsymbol{\alpha}_{i}\right]_{s_{j}}^{2}=\left(\mathbf{q}_{i}-\mathbf{\Phi} \boldsymbol{\alpha}_{i}\right)^{T}\left(\mathbf{q}_{i}-\mathbf{\Phi} \boldsymbol{\alpha}_{i}\right) .
$$

where the notation $[\cdot]_{j}$ is used to denote the $j$-th components of a vector. Expression (11) is proportional to the negative log-likelihood of the data for a linear statistical model of the form:

$$
\mathbf{q}_{i}=\boldsymbol{\Phi} \boldsymbol{\alpha}_{i}+\epsilon_{i}
$$

with normally distributed error vector $\boldsymbol{\epsilon}_{i} \sim \mathcal{N}\left(\mathbf{0}, \sigma^{2} I\right)$, and likelihood $\ell_{\boldsymbol{\alpha}_{i}}\left(\mathbf{q}_{i}\right)=\mathcal{N}\left(\boldsymbol{\Phi} \boldsymbol{\alpha}_{i}, \sigma^{2} I\right)$. The quantity $\mathbf{C}_{q_{i}}=\sigma^{2} I$ is used in what follows to denote the covariance of the flow rate at $\Gamma_{i}$. The value of $\boldsymbol{\alpha}_{i}$ determined by minimizing (11) is generally referred to as the OLS estimate of the coefficient vector, is unbiased, and achieves asymptotic efficiency with covariance that attains the Cramér-Rao bound (see, e.g., Banks et al., 
2014). Under these conditions, the inverse of the Fisher information matrix evaluated at the optimal estimate $\boldsymbol{\alpha}_{i}=\boldsymbol{\alpha}_{i, M L E}$ (see, e.g., Rothenberg, 1971) is used to quantify the second order statistics of $\boldsymbol{\alpha}_{i, M L E}$. This takes the form (note that, in the interest of clarity, we drop the index $i$ in $\boldsymbol{\alpha}_{i}$ ):

$$
\mathcal{I}(\boldsymbol{\alpha})=\mathbb{E}\left[\left(\frac{\partial \log \ell_{\boldsymbol{\alpha}}(\mathbf{q})}{\partial \boldsymbol{\alpha}}\right)^{T}\left(\frac{\partial \log \ell_{\boldsymbol{\alpha}}(\mathbf{q})}{\partial \boldsymbol{\alpha}}\right)\right]=-\mathbb{E}\left[\frac{\partial^{2} \log \ell_{\boldsymbol{\alpha}}(\mathbf{q})}{\partial \boldsymbol{\alpha} \partial \boldsymbol{\alpha}^{T}}\right]=\frac{1}{\sigma^{2}} \boldsymbol{\Phi}^{T} \boldsymbol{\Phi} .
$$

and $\operatorname{Cov}\left(\boldsymbol{\alpha}_{M L E}\right)$, the covariance matrix of the coefficient vector $\boldsymbol{\alpha}$ at $\boldsymbol{\alpha}_{M L E}$ is estimated as $\mathcal{I}^{-1}\left(\boldsymbol{\alpha}_{M L E}\right)$.

\subsection{RVM estimator}

Relevance vector machines, introduced in Tipping (2001), Tipping et al. (2003), combine Bayesian estimation with sparsity promoting priors, providing simultaneous computation of compact representations and associated uncertainty. Hyperpriors are specified over each coefficient $\alpha_{j, i}$ in (12), consisting of independent, zero-mean, Gaussian components with precision vector $\boldsymbol{\beta}=\left(\beta_{1}, \beta_{2}, \ldots, \beta_{n_{b}}\right)$, i.e.:

$$
P\left(\boldsymbol{\alpha}_{i} \mid \boldsymbol{\beta}\right)=\frac{1}{(2 \pi)^{n_{b} / 2} \prod_{k=1}^{n_{b}} \beta_{k}} \exp \left[-\frac{1}{2} \sum_{j=1}^{n_{b}} \beta_{j} \alpha_{j, i}^{2}\right]=\frac{1}{(2 \pi)^{n_{b} / 2} \prod_{k=1}^{n_{b}} \beta_{k}} \exp \left[-\frac{1}{2} \boldsymbol{\alpha}_{i}^{T} \mathbf{Z} \boldsymbol{\alpha}_{i}\right]
$$

with $\mathbf{Z}$ being a diagonal matrix with $Z_{j, j}=\beta_{j}$. The posterior $P\left(\boldsymbol{\alpha}_{i} \mid \mathbf{q}_{i}, \boldsymbol{\beta}, \sigma^{2}\right) \sim \mathcal{N}(\boldsymbol{\mu}, \boldsymbol{\Sigma})$ is also Gaussian. Using the Bayes' rule and completing the squares, one obtains:

$$
\boldsymbol{\mu}=\sigma^{-2} \boldsymbol{\Sigma} \boldsymbol{\Phi} \mathbf{q}_{i}, \quad \boldsymbol{\Sigma}=\left(\boldsymbol{Z}+\sigma^{-2} \boldsymbol{\Phi}^{T} \boldsymbol{\Phi}\right)^{-1} .
$$

Hyperparameters are estimated as part of the regression strategy, following an approach referred to as typeII maximum likelihood or empirical Bayes estimation (see, e.g., Berger, 1985, Carlin and Louis, 1997). In practice, values of $\beta_{j}$ are determined by iterative maximization of the marginal log-likelihood

$$
\mathcal{M}(\boldsymbol{\beta})=\log P\left(\mathbf{q}_{i} \mid \boldsymbol{\beta}, \sigma^{2}\right)=-\frac{1}{2}\left[N \log (2 \pi)+\log |\mathbf{C}|+\mathbf{q}_{i}^{T} \mathbf{C}^{-1} \mathbf{q}_{i}\right],
$$

where $\mathbf{C}=\sigma^{2} \mathbf{I}+\boldsymbol{\Phi} \mathbf{Z}^{-1} \boldsymbol{\Phi}^{T}$. A key observation is that (16) can be decomposed as $\mathcal{M}(\boldsymbol{\beta})=\mathcal{M}\left(\boldsymbol{\beta}_{-j}\right)+m\left(\beta_{j}\right)$ i.e., the marginal likelihood obtained by assigning $\alpha_{j, i}=0$, and its increment produced by including the $j$-th basis function in the model (i.e., the set of basis functions with non zero expansion coefficient). By algebraic manipulations, it can be shown that the increment in marginal likelihood for the generic basis function depends mainly upon two quantities,

$$
f_{q, j}=\phi_{j}^{T} \mathbf{C}_{-j}^{-1} \mathbf{q}_{i}, \quad f_{s, j}=\phi_{j}^{T} \mathbf{C}_{-j}^{-1} \phi_{j}
$$

where $f_{s, j}$, the sparsity factor, quantifies how much the selected basis vector is similar to other vectors already included in the model, and $f_{q, j}$, the quality factor, is a measure of its correlation with the residual at the current regression iteration. These factors are defined as external quality and sparsity factors for a given basis, as they are computed without including the contribution of the associated basis in $\mathbf{C}$. We also define $\theta_{j}=f_{q, j}^{2}-f_{s, j}$ and look at the sign of this quantity to decide whether to add, remove or re-estimate the selected basis coefficient, based on the choice that maximally increases the marginal likelihood (16).

At every iteration, the covariance $\boldsymbol{\Sigma}$, average vector $\boldsymbol{\mu}$ and internal quality and sparsity factor vectors $\mathbf{F}_{q}$ and $\mathbf{F}_{s}$ need to be updated. While fast evaluation of these updates is possible for a fixed noise variance $\sigma^{2}$ as discussed in Tipping et al. (2003), an effective reduction of the iteration residual is obtained by re-estimation of $\sigma^{2}$ at every iteration. The approximation algorithm therefore proceeds with alternate estimations of the coefficient vector $\boldsymbol{\mu}$ and noise variance. An updated noise variance, leading to maximization of (16) can also be estimated (see, Tipping, 2001) as follows:

$$
\sigma^{2}=\frac{\left\|\mathbf{q}_{i}-\boldsymbol{\Phi} \boldsymbol{\alpha}_{i}\right\|^{2}}{N-M+\sum_{j} \beta_{j} \boldsymbol{\Sigma}_{j j}}
$$


The inverse covariance matrix $\boldsymbol{\Sigma}^{-1}$ is computed after Cholesky factorization $\boldsymbol{\Sigma}^{-1}=\mathbf{L} \mathbf{L}^{T}$. Typically, the reduced size of $\boldsymbol{\Sigma}^{-1}$ follows from the assumption of sparsity in the representation $\boldsymbol{\alpha}_{i}$ and therefore this matrix inversion can be carried out at relatively low computational cost. The average coefficient vector $\boldsymbol{\mu}=\sigma^{-2} \boldsymbol{\Sigma} \boldsymbol{\Phi}^{T} \mathbf{u}$ is updated, followed by computation of the internal sparsity and quality vectors

$$
\begin{aligned}
& F_{s, j}=\phi_{j}^{T} \mathbf{B} \phi_{j}-\phi_{j}^{T} \mathbf{B} \boldsymbol{\Phi} \Sigma \boldsymbol{\Phi}^{T} \mathbf{B} \phi_{j} \\
& F_{q, j}=\phi_{j}^{T} \mathbf{B} \mathbf{q}_{i}-\phi_{j}^{T} \mathbf{B} \boldsymbol{\Phi} \Sigma \boldsymbol{\Phi}^{T} \mathbf{B} \mathbf{q}_{i},
\end{aligned}
$$

where $\mathbf{B}=\sigma^{-2} \mathbf{I}$. External factors $f_{s, j}$ and $f_{q, j}$ are finally computed using the expression

$$
f_{s, j}=\frac{\beta_{j} F_{s, j}}{\beta_{j}-F_{s, j}}, \quad \text { and similarly, } \quad f_{q, j}=\frac{\beta_{j} F_{q, j}}{\beta_{j}-F_{s, j}} .
$$

We conclude this section by highlighting why we choose RVMs over other widely used families of algorithms for sparse regression. A comparison between RVMs and Orthogonal Matching Pursuit (OMP) heuristic (see, Tropp et al., 2007), for example, leads to the following observations. During the OMP iterations, a basis function can only be added to the model based on its correlation with the residual vector at the current iteration. More flexibility is provided by RVMs in this regard, where basis functions can be added to or removed from the model or the associated representation coefficient re-estimated. Moreover, simultaneous estimation of regression coefficients and noise intensity cannot be performed with OMP but cross validationbased approaches can be used at a higher computational cost. RVMs naturally deal with this problem by alternating estimations of the coefficient vector and its covariance with re-estimation of the noise intensity. Note however that, for variable noise intensity, fast update of the iteration quantities is not possible and one has to deal with matrix inversion operations whose computational cost increases considerably with the number of basis included in the model. One of the main advantages of RVM over OMP is the ability of estimating the full covariance matrix of $\boldsymbol{\alpha}_{i}$, allowing to associate measures of confidence to the computed expansions.

Finally, we remark how the adopted hyper-priors for $\boldsymbol{\alpha}_{i}$ are zero-mean Gaussian distributions, and therefore sparsity in the regression coefficients is promoted by RVM, meaning that sparser expansion coefficient vectors lead to higher values of the marginal likelihood. We also stress that sparsity in this case means that the flow rate at the generic $\Gamma_{i}$ depends on the average pressure at only a limited number of other outlets.

Remark 1 (Posterior predictive distribution for inlet/outlet flow rate). Multivariate Gaussian dis tributions for the expansion coefficients $\boldsymbol{\alpha}_{i}$ at $\Gamma_{i}$ are identified through MLE or RVM in terms of an average coefficient estimate $\hat{\boldsymbol{\alpha}}_{i}$ and associated covariance $\boldsymbol{C}_{\hat{\alpha}}$. Due to the linearity in (12), it follows that $\mathbf{q}_{i} \sim \mathcal{N}\left(\boldsymbol{\Phi} \hat{\boldsymbol{\alpha}}, \sigma^{2} \mathbf{I}+\boldsymbol{\Phi} \boldsymbol{C}_{\hat{\alpha}} \boldsymbol{\Phi}^{T}\right)$.

\section{Construction of pressure-flow surrogates for patient specific models}

In this section, we compare the performance of OLS and RVM in approximating flow rates from pressures at each $\Gamma_{i}$. We have chosen two multiscale models representative of the open loop and closed loop boundary condition families, though the method is generally applicable. We will focus, in particular, on the agreement between the flow rates produced at every $\Gamma_{i}$ by the reference multiscale solution and the computed reconstructions. Note that all reconstructions shown in the following sections adopt a simple linear relationship between pressures and flow rates at the interfaces.

\subsection{Abdominal aortic model with open loop boundary conditions}

We first consider a model of the abdominal aorta with boundary conditions provided by RCR blocks and prescribed inlet flow rate (Figure 4). The incompressible Navier-Stokes equations were solved using the open source package SimVascular (Whiting and Jansen, 2001, Wilson et al., 2001) for 7 heart cycles, using 
a timestep of $7.5 \times 10^{-3} \mathrm{~s}$ under uniform blood density $1.06 \mathrm{~g} \cdot \mathrm{cm}^{-3}$ and viscosity $0.04 \mathrm{gr} / \mathrm{cm} / \mathrm{s}$. The threedimensional SUPG finite element solver was implicitly coupled with RCR blocks at the 19 outlets (a typical RCR layout is illustrated in Figure 6b, the selected parameter set is reported in Table 2). The flow rate time history shown in Figure 6a was prescribed at the aortic inlet using a Womerseley flow profile (Womersley, 1955). Flow rate reconstructions at $\Gamma_{i}$, performed using both OLS and RVM are illustrated in Figures 6. The shaded areas in the graph show $95 \%$ confidence intervals associated with the computed reconstructions determined using an amplification of the predictive variance. Reconstructions were performed under three different scenarios characterized by a sampling ratio $m /\left(m_{t}-m_{w}\right)$ equal to $0.05,0.2$ and 0.8 , respecitvely, but, due to qualitative similarity, OLS and RVM approximants are only shown for $m /\left(m_{t}-m_{w}\right)=0.8$ in Figure 6. Average flow rates reconstructions were very similar for these scenarios, the main difference being the $95 \%$ confidence interval computed through RVM, as shown in Figure 6 for the Inferior Mesenteric Artery (IMA) and left Superior Renal Artery. OLS reconstructions seem affected by limited uncertainty, compared to those generated using RVM, in particular at the outlets associated with limited flow rate. Finally, Figure 7 shows the distributions of the approximation residuals at each outlet, whose average value is computed as

$$
\bar{r}_{i}=\frac{1}{m} \sum_{j=1}^{m}\left[Q_{s_{j}, i}-\mathbf{\Phi}\left(P_{s_{j}, 1}, P_{s_{j}, 2}, \ldots, P_{s_{j}, d}\right) \boldsymbol{\alpha}_{i}\right]
$$

confirming the validity of the assumption of a Gaussian independent error vector of zero mean made in the derivation of (12).

\subsection{Coronary circulation model with closed loop boundary conditions}

A multiscale coronary circulation model with bypass grafts is constructed based on Sankaran et al. (2012) and shown in Figure 9. These parameters were selected using manual tuning to have a satisfactory agreement with clinical targets, including $\mathrm{max} / \mathrm{min}$ aortic blood pressure, stroke volume, Aorta/Coronary blood flow split and flow split between right and left coronaries. Atrial and ventricular pressure-volume (PV) relationships are modeled using an activation and elastance formulation, respectively (Senzaki et al., 1996, Corsini et al., 2014). The nomenclature used for the model outlets is illustrated in Figure 8. Multiscale flow waveforms and regressions computed through OLS and RVM are shown in Figure 10, at four selected interfaces, aortic outlet, first aortic branch, first left coronary artery outlet and fourth right coronary artery outlet. Due to qualitative similarity, OLS and RVM approximants were computed for several sampling ratios $(0.05,0.2$ and 0.8$)$ but are only shown for $m /\left(m_{t}-m_{w}\right)=0.8$. Finally, approximation residuals are shown in Figure 11.

\subsection{Integration of $O L S$ and $R V M$ approximants in $L P N$ circulation networks}

Integration of surrogate models into ODE solvers for circulation networks (a schematic idea of this is shown in Figure 9) leads to very different results for the two regression approaches. The adopted numerical approximation scheme ( $4^{\text {th }}$ order Runge-Kutta approach with constant time step), rapidly diverges if coupled with an OLS surrogate, while the RVM approach leads to working models and satisfactory agreement in both cases. While the reason behind the failure of surrogates generated through OLS will be further discussed in Section 5, only RVM results will be considered in this section.

Once again we remind the reader that we truncated the polynomial approximant that mimics the relationship between interface pressure-flow rates at the first order and that stable RVM surrogates are obtained by training with a relatively small number of samples, i.e., $m /\left(m_{t}-m_{w}\right)=0.05$. While it could be argued that the assumption of proportionality between pressure drop and the flow rate does not hold for vessels with concentrated stenosis and/or significant tapering, our results show that a relationship directly in terms of average pressures and flow rates at the model interfaces provides a satisfactory approximation of the multiscale model behavior even for geometries containing a wide range of vessel diameters and a time-varying flow rate. A possible explanation relates to the fact that the vessel flow rate is not independent on its diameter in our models. This means that the significant resistance offered by small diameter vessels triggers 
a drop in their flow rate, that, in turn, allows the pressure/flow rate relationship to be well approximated by a linear regime.

Results comparing use of manually tuned resistances and the proposed RVM approximant are reported in Figures 12, 13 and 14 for the Coronary artery model. In these figures, the Manual label refers to an alternative surrogate model that was computed by uniformly scaling the resistances of the RC blocks at each outlet. This scaling factor was determined via manual iterations in order to achieve satisfactory approximations of multiscale pressure and flow rate time histories. Using these manually tuned resistances, flow rates at the generic $\Gamma_{i}$ interface were determined as $q_{i}=R_{i}\left(p_{\text {inlet }}-p_{i}\right)$, where $p_{\text {inlet }}$ is the pressure at the aortic inlet and $R_{i}$ the associated outlet resistance.

Finally, results for the model of the abdominal aorta are reported in Figure 15. Interface pressures and flow rates in the multiscale model are in good agreement with those obtained from a lumped parameter network model that includes the proposed surrogate both for the abdominal aorta and the coronary circulation cases.

\section{Features of computed expansion coefficients}

In this section, we focus on possible manipulations of the expansion coefficients determined through OLS and RVM regression. We first discuss the possibility of computing the global sensitivity indexes for the flow rates, to better understand the hierarchy of outlets in the model, i.e., those whose variation in pressure are more likely to affect the distribution of blood flowing through the model. Second, we start from the regression coefficients to compute how pressure perturbations are amplified or reduced by the two families of polynomial regressors.

\subsection{Sobol decomposition of the flow rate variance}

In this section, we consider a Sobol' decomposition (Sobol', 2001) of the normalized pressure-flow rate relation $q_{i}=G(\mathbf{x})$ in terms of zero mean contributions of increasing dimensionality

$$
\begin{aligned}
q_{i}\left(x_{1}, \ldots, x_{n}\right)= & G_{0}+\sum_{j=1}^{n} G_{j}\left(x_{j}\right)+\sum_{j=1}^{n-1} \sum_{k=j+1}^{n} G_{j, k}\left(x_{j}, x_{k}\right)+\cdots+G_{1, \ldots, n}\left(x_{1}, \ldots, x_{d}\right), \text { where } \\
& \int_{[-1,1]} G_{j_{1}, \ldots, j_{l}}\left(x_{j_{1}}, \ldots, x_{j_{l}}\right) d y_{j_{k}}=0, \forall 1 \leq k \leq l, \forall\left\{j_{1}, \ldots, j_{l}\right\} \subset\{1, \ldots, d\} .
\end{aligned}
$$

Under these assumptions, $G_{0}$ represents the mean of $G$, while its variance becomes:

$$
\mathbb{V}[G(\mathbf{x})]=\sigma^{2}=\int_{[-1,1]^{n}} G(\mathbf{x})^{2} d \mathbf{x}-G_{0}^{2}=\sum_{j=1}^{n} \sigma_{j}^{2}+\sum_{j=1}^{n-1} \sum_{k=j+1}^{n} \sigma_{j, k}^{2}+\cdots+\sigma_{1, \ldots, n}^{2},
$$

as products of terms in (22) are all zero. This leads to a decomposition of the total variance of $G$ in single parameter effects, combined effects of two parameters, three parameters and so on. The combined global effect of the parameter set $\left\{x_{j_{1}}, \ldots, x_{j_{l}}\right\}$ can therefore be quantified using the following direct sensitivity index

$$
S_{x_{j_{1}}, \ldots, x_{j_{l}}}=\frac{\sigma_{j_{1}, \ldots, j_{l}}^{2}}{\sigma^{2}}, \quad \text { where, } \quad \sum_{j=1}^{n} S_{j}+\sum_{j=1}^{n-1} \sum_{k=j+1}^{n} S_{j, k}+\cdots+S_{1, \ldots, n}=1 .
$$

At this point we note that the Legendre expansion in (3) represents a Sobol' decomposition of $q_{i}$ with the additional property that it is possible to separate the effects associated to the various polynomial orders. In this study, the global sensitivity indices accounting for the direct effects of the parameter $x_{i}$ are computed using

$$
S_{x_{i}}=\frac{\sum_{j \in J} \alpha_{j, i}^{2}}{\sum_{j=1}^{p} \alpha_{j, i}^{2}}
$$


where the set $J$ contains the multi-indexes with non zero $x_{i}$ component. Finally, we note that direct and total sensitivities are equal for linear approximation models. Figure 16 and 17 show the sensitivity matrices for OLS and RVM regression in the two selected models. The entries in each row have sum equal to $100 \%$ and quantify the effect of the outlet listed in the corresponding column.

We observe that the importance of the aortic outlet pressure and aortic inlet pressure is emphasized by sparse regression for both the coronary and abdominal aortic models. This is expected, as pressure variations at major inlet and outlets in the models should significantly affect the flow rate response of minor vessels. For the coronary model other important outlets are highlighted, such as Lca5, due to presence of a coronary bypass graft and Rca3 which is the main outlet of the right coronary artery.

The proposed analysis also suggests a possible use of numerical models to understand how the flow rate at a specific branch is affected by the pressure in the other vessels. Therefore non-local (i.e., at different branches) alterations of pressure may be pursued as an alternative strategy to improve the flow rate at a specific branch or, for example, to design optimal locations of bypass grafts.

The difference between sensitivity plots computed through OLS and RVM regression is striking, and highlights the benefits of sparse regression in recognizing the main interfaces whose average pressure significantly affects the flow rates at the other outlets. OLS sensitivities look much more diffused over the outlets as the only goal is residual minimization and therefore a significant contribution of few outlets may be equivalent to a smaller contribution of a greater number of them.

\subsection{Stability of $O L S$ and RVM surrogates}

In this section, we investigate why sparsity promoting approximations produce stable surrogates of threedimensional physiological models while least squares approaches fail to do so. The notion of stability, in this case, is used informally for surrogates that produce working lumped parameter network models (with pressure and flow rate time histories reaching stationarity after a few simulated heart cycles).

A more formal treatment of stability considers how outlet flow rates are amplified by the surrogate as a result of pressure perturbations. We consider, in particular, a Gaussian pressure noise of uniform variance $\sigma^{2}$ and flow rates at the interface written as a linear combination of outlet pressures, as follows:

$$
q_{i}=\sum_{j=2}^{n+1} \alpha_{j, i} p_{j}=\hat{\boldsymbol{\alpha}}_{i} \boldsymbol{p}, \text { and } \quad \boldsymbol{q}=\boldsymbol{A} \boldsymbol{p}
$$

and note that a pressure perturbation $\delta \mathbf{p} \sim \mathcal{N}\left(\mathbf{0}, \sigma^{2} \mathbf{I}\right)$ will generate a flow rate perturbation $\delta \mathbf{q} \sim$ $\mathcal{N}\left(\mathbf{0}, \sigma^{2} \mathbf{A} \mathbf{A}^{T}\right)$. The eigenvalues of $\mathbf{A} \mathbf{A}^{T}$ thus represent the noise amplification factors through the linear transformation expressed by A. Figure 1 illustrates the eigenvalue spectra for the coefficient representations obtained through OLS and RVM regression for the two models of the coronary and abdominal aortic circulation. The figure shows that OLS regression produces significantly larger amplification factors than RVM and therefore it is likely for surrogate models produced by this technique to be less stable when included in ODE circulation models as shown in Section 4.3.

\section{Conclusion}

In this study, we have presented a methodology to build a reduced order representation of a threedimensional hemodynamic finite element model, using sparse polynomial regression on the pressure/flow rate relationship at the interface with peripheral lumped parameter models.

Accurate reconstructions for these quantities can be obtained, in rigid cardiovascular models, using a simple linear pressure-flow rate relationship, disregarding blood inertia and time dependence.

We have shown that while OLS regression results in smaller approximation errors, surrogates are generally not stable, and therefore cannot reliably be included in multiscale simulations. This can be explained, at least in part, by their tendency to over-amplify outlet pressure perturbations. The preferential dependence 


\begin{tabular}{|c|c|c|c|c|c|}
\hline$i$ & $\Gamma_{i}$ & $R_{p}[\mathrm{Ba} \cdot \mathrm{s} / \mathrm{mL}]$ & $C[\mathrm{~mL} / \mathrm{Ba}]$ & $R_{d}[\mathrm{Ba} \cdot \mathrm{s} / \mathrm{mL}]$ & $p_{d} \mid$ \\
\hline 1 & Inferior mesenteric artery & 1583.13 & $3.44 \times 10^{-5}$ & 34215.40 & 0.0 \\
2 & Right interior iliac artery & 3169.51 & $1.72 \times 10^{-5}$ & 70363.10 & 0.0 \\
3 & Right interior iliac artery branch 1 & 7094.64 & $7.7 \times 10^{-6}$ & 157504.00 & 0.0 \\
4 & Right interior iliac artery branch 2 & 7094.64 & $7.7 \times 10^{-6}$ & 157504.00 & 0.0 \\
5 & Left interior iliac artery branch 1 & 7094.64 & $7.7 \times 10^{-6}$ & 157504.00 & 0.0 \\
6 & Left interior iliac artery & 3903.05 & $1.40 \times 10^{-5}$ & 86649.70 & 0.0 \\
7 & Left interior iliac artery branch 2 & 7094.64 & $7.7 \times 10^{-6}$ & 157504.00 & 0.0 \\
8 & Right exterior iliac artery & 718.27 & $7.60 \times 10^{-5}$ & 15945.20 & 0.0 \\
9 & Left exterior iliac artery & 718.27 & $7.60 \times 10^{-5}$ & 15945.20 & 0.0 \\
10 & Superior mesenteric artery & 867.84 & $6.27 \times 10^{-5}$ & 18429.90 & 0.0 \\
11 & Left inferior renal artery & 829.38 & $6.56 \times 10^{-5}$ & 17593.90 & 0.0 \\
12 & Left superior renal artery & 1330.80 & $4.08 \times 10^{-5}$ & 27260.40 & 0.0 \\
13 & Superior mesenteric artery branch 1 & 1617.35 & $3.37 \times 10^{-5}$ & 35259.30 & 0.0 \\
14 & Right superior renal artery & 609.70 & $8.93 \times 10^{-5}$ & 12991.00 & 0.0 \\
15 & Right inferior renal artery & 2222.74 & $2.45 \times 10^{-5}$ & 47359.60 & 0.0 \\
16 & Splenic artery branch 1 & 2096.00 & $2.60 \times 10^{-5}$ & 45000.00 & 0.0 \\
17 & Splenic artery branch 2 & 2096.00 & $2.60 \times 10^{-5}$ & 45000.00 & 0.0 \\
18 & Hepatic artery branch 2 & 2096.00 & $2.60 \times 10^{-5}$ & 45000.00 & 0.0 \\
19 & Hepatic artery branch 1 & 2096.00 & $2.60 \times 10^{-5}$ & 45000.00 & 0.0 \\
\hline
\end{tabular}

Table 2: Characterization of boundary RCR circulation blocks at every interface $\Gamma_{i}$.

between flow rate and pressure at the outlets is instead better captured by RVM regression, promoting sparsity in the resulting coefficient representation. Furthermore, stable surrogates are obtained using RVM offering comparable accuracy in representing the original multiscale model response than optimally tuned resistance assemblies.

Finally, use of orthogonal polynomial expansions allows a straightforward computation of outlet sensitivities giving a practical tool to quantify interactions between outlet flow rates and pressures. This approach shows promise for significantly reducing computational cost in cardiovascular blood flow simulation problems requiring multiple function evaluations such as tuning, optimization, and uncertainty quantification.

Future studies will be devoted to demonstrating utility of these models in automated tuning and other parameter estimation and optimization applications in a range of cardiovascular blood flow simulation applications.

\section{Acknowledgements}

The authors would like to thank the three anonymous Reviewers for their generous comments and feedback that greatly contributed to improve the consistency and quality of the present contribution. The authors would also like to thank Justin Tran for his contributions to the coronary LPN model code and Abhay Bangalore Ramachandra for his work on the lumped parameter formulation of the same model. This work was supported by American Heart Association Grant \#15POST23010012 (Daniele Schiavazzi) and by the Leducq Foundation as part of a Transatlantic Network of Excellence for Cardiovascular Research, a Burroughs Wellcome Fund Career award at the Scientific Interface, NSF CAREER OCI-1150184 and NIH R01HL123689 (Alison Marsden) and used computational resources from the Extreme Science and Engineering Discovery Environment (XSEDE), supported by National Science Foundation grant number ACI-1053575. We also acknowledge the open source SimVascular project at www.simvascular.org. 


\section{References}

Antoulas, A., Beattie, C., Gugercin, S., 2010. Interpolatory model reduction of large-scale dynamical systems. In: Efficient Modeling and Control of Large-Scale Systems. Springer, pp. 3-58.

Avanzolini, G., Barbini, P., Cappello, A., 1992. Comparison of algorithms for tracking short-term changes in arterial circulation parameters. Biomedical Engineering, IEEE Transactions on 39 (8), 861-867.

Ballarin, F., 2015. Reduced-order models for patient-specific haemodynamics of coronary artery bypass grafts. Ph.D. thesis, Politecnico di Milano.

Banks, H., Hu, S., Thompson, W., 2014. Modeling and Inverse Problems in the Presence of Uncertainty. CRC Press.

Benner, P., Gugercin, S., Willcox, K., 2013. A survey of model reduction methods for parametric systems.

Berger, J., 1985. Statistical decision theory and Bayesian analysis. Springer Science \& Business Media.

Berkooz, G., Holmes, P., Lumley, J., 1993. The proper orthogonal decomposition in the analysis of turbulent flows. Annual Review of Fluid Mechanics 25 (1), 539-575.

Carlin, B., Louis, T., 1997. Bayes and empirical Bayes methods for data analysis. Statistics and Computing 7 (2), $153-154$.

Chen, P., Quarteroni, A., Rozza, G., 2015. Reduced order methods for uncertainty quantification problems.

Clark, J., Ling, R., Srinivasan, R., Cole, J., Pruett, R., 1980. A two-stage identification scheme for the determination of the parameters of a model of left heart and systemic circulation. Biomedical Engineering, IEEE Transactions on (1), 20-29.

Colciago, C. M., 2014. Reduced Order Fluid-Structure Interaction Models for Haemodynamics Applications. Ph.D. thesis, SB, Lausanne.

Corsini, C., Baker, C., Kung, E., Schievano, S., Arbia, G., Baretta, A., Biglino, G., Migliavacca, F., Dubini, G., Pennati, G., et al., 2014. An integrated approach to patient-specific predictive modeling for single ventricle heart palliation. Computer Methods in Biomechanics and Biomedical Engineering 17 (14), 1572-1589.

Deswysen, B., 1977. Parameter estimation of a simple model of the left ventricle and of the systemic vascular bed, with particular attention to the physical meaning of the left ventricular parameters. Biomedical Engineering, IEEE Transactions on (1), 29-38.

Deswysen, B., Charlier, A., Gevers, M., 1980. Quantitative evaluation of the systemic arterial bed by parameter estimation of a simple model. Medical and Biological Engineering and Computing 18 (2), 153-166.

Dotter, C., Roberts, D., Steinberg, I., 1950. Aortic length: angiocardiographic measurements. Circulation 2 (6), $915-920$.

Ferranti, F., Tamburrelli, V., Antonini, G., 2015. Rational macromodeling of 1D blood flow in the human cardiovascular system. International Journal for Numerical Methods in Biomedical Engineering 31 (3).

Formaggia, L., Gerbeau, J., Nobile, F., Quarteroni, A., 2001. On the coupling of 3D and 1D Navier-Stokes equations for flow problems in compliant vessels. Computer Methods in Applied Mechanics and Engineering 191 (6), 561-582.

Gugercin, S., Antoulas, A., 2004. A survey of model reduction by balanced truncation and some new results. International Journal of Control 77 (8), 748-766.

Guibert, R., Mcleod, K., Caiazzo, A., Mansi, T., Fernández, M., Sermesant, M., Pennec, X., Vignon-Clementel, I., Boudjemline, Y., Gerbeau, J., 2014. Group-wise construction of reduced models for understanding and characterization of pulmonary blood flows from medical images. Medical Image Analysis 18 (1), 63-82.

Hager, A., Kaemmerer, H., Rapp-Bernhardt, U., Blücher, S., Rappa, K., Bernhardt, T., Galanski, M., Hess, J., 2002. Diameters of the thoracic aorta throughout life as measured with helical computed tomography. The Journal of Thoracic and Cardiovascular Surgery 123 (6), 1060-1066.

McInnis, B., Guo, Z.-W., Lu, P., Wang, J.-C., 1985. Adaptive control of left ventricular bypass assist devices. Automatic Control, IEEE Transactions on 30 (4), 322-329.

McLeod, K., Caiazzo, A., Fernández, M., Mansi, T., Vignon-Clementel, I., Sermesant, M., Pennec, X., Boudjemline, Y., Gerbeau, J., 2010. Atlas-based reduced models of blood flows for fast patient-specific simulations. In: Statistical Atlases and Computational Models of the Heart. Springer, pp. 95-104.

Milišić, V., Quarteroni, A., 2004. Analysis of lumped parameter models for blood flow simulations and their relation with 1D models. ESAIM: Mathematical Modeling and Numerical Analysis 38 (04), 613-632.

Moghadam, M., Vignon-Clementel, I., Figliola, R., Marsden, A., (mocha) Investigators, M. O. C. H. A., et al., 2013. A modular numerical method for implicit 0D/3D coupling in cardiovascular finite element simulations. Journal of Computational Physics $244,63-79$.

Revie, J. A., Stevenson, D. J., Chase, J. G., Hann, C. E., Lambermont, B. C., Ghuysen, A., Kolh, P., Shaw, G. M., Heldmann, S., Desaive, T., 2013. Validation of subject-specific cardiovascular system models from porcine measurements. Computer Methods and Programs in Biomedicine 109 (2), 197-210.

Rothenberg, T., 1971. Identification in parametric models. Econometrica: Journal of the Econometric Society, $577-591$.

Rowley, C., 2005. Model reduction for fluids, using balanced proper orthogonal decomposition. International Journal of Bifurcation and Chaos 15 (03), 997-1013.

Rozza, G., Huynh, D., Patera, A., 2008. Reduced basis approximation and a posteriori error estimation for affinely parametrized elliptic coercive partial differential equations. Archives of Computational Methods in Engineering 15 (3), $229-275$.

Ruchti, T. L., Brown, R. H., Jeutter, D. C., Feng, X., 1993. Identification algorithm for systemic arterial parameters with application to total artificial heart control. Annals of biomedical engineering 21 (3), 221-236.

Sankaran, S., Moghadam, M., Kahn, A., Tseng, E., Guccione, J., Marsden, A., 2012. Patient-specific multiscale modeling of blood flow for coronary artery bypass graft surgery. Annals of Biomedical Engineering 40 (10), 2228-2242.

Senzaki, H., Chen, C., Kass, D., 1996. Single-beat estimation of end-systolic pressure-volume relation in humans a new method with the potential for noninvasive application. Circulation 94 (10), 2497-2506. 
Shimooka, T., Mitamura, Y., Yuhta, T., 1991. Investigation of parameter estimator and adaptive controller for assist pump by computer simulation. Artificial organs 15 (2), 119-128.

Sobol', I., 2001. Global sensitivity indices for nonlinear mathematical models and their Monte Carlo estimates. Mathematics and Computers in Simulation 55 (1), 271-280.

Spilker, R., Taylor, C., 2010. Tuning multidomain hemodynamic simulations to match physiological measurements. Annals of Biomedical Engineering 38 (8), 2635-2648.

Sughimoto, K., Liang, F., Takahara, Y., Mogi, K., Yamazaki, K., Takagi, S., Liu, H., 2013. Assessment of cardiovascular function by combining clinical data with a computational model of the cardiovascular system. The Journal of Thoracic and Cardiovascular Surgery 145 (5), 1367-1372.

Tipping, M., 2001. Sparse bayesian learning and the relevance vector machine. The Journal of Machine Learning Research 1, $211-244$.

Tipping, M., Faul, A., et al., 2003. Fast marginal likelihood maximisation for sparse Bayesian models. In: Proceedings of the ninth International Workshop on Artificial Intelligence and Statistics. Vol. 1.

Tropp, J., Gilbert, A., et al., 2007. Signal recovery from random measurements via orthogonal matching pursuit. Information Theory, IEEE Transactions on 53 (12), 4655-4666.

Vignon-Clementel, I., Figueroa, C., Jansen, K., Taylor, C., 2006. Outflow boundary conditions for three-dimensional finite element modeling of blood flow and pressure in arteries. Computer Methods in Applied Mechanics and Engineering 195 (29), 3776-3796.

Waller, B., Orr, C., Slack, J., Pinkerton, C., Van Tassel, J., Peters, T., 1992. Anatomy, histology, and pathology of coronary arteries: A review relevant to new interventional and imaging techniques part i,ii,iii,iv. Clinical Cardiology 15 (6), $451-457$.

Whiting, C., Jansen, K., 2001. A stabilized finite element method for the incompressible Navier-Stokes equations using a hierarchical basis. International Journal for Numerical Methods in Fluids 35 (1), 93-116.

Wilson, N., Wang, K., Dutton, R., Taylor, C., 2001. A software framework for creating patient specific geometric models from medical imaging data for simulation based medical planning of vascular surgery. In: Medical Image Computing and Computer-Assisted Intervention-MICCAI 2001. Springer, pp. 449-456.

Womersley, J., 1955. Method for the calculation of velocity, rate of flow and viscous drag in arteries when the pressure gradient is known. The Journal of Physiology 127 (3), 553-563.

Xiao, X., Ozawa, E., Huang, Y., Kamm, R., 2002. Model-based assessment of cardiovascular health from noninvasive measurements. Annals of Biomedical Engineering 30 (5), 612-623.

Yu, Y., Boston, J., Simaan, M., Antaki, J., 2001. Minimally invasive estimation of systemic vascular parameters. Annals of Biomedical Engineering 29 (7), 595-606.

Yu, Y.-C., Boston, J. R., Simaan, M. A., Antaki, J. F., 1998. Estimation of systemic vascular bed parameters for artificial heart control. Automatic Control, IEEE Transactions on 43 (6), 765-778. 


\title{
On a sparse pressure-flow rate condensation of rigid circulation models
}

\author{
D. E. Schiavazzi, T. Y. Hsia and A. L. Marsden
}

Figure and Table Captions

Figure 1: Schematic illustration of the series arrangment of inductance and resistance lumped elements in one ideal cylindrical vessel.

Figure 2: Inertance and resistance contribution of to the total pressure drop, for the scenarios illustrated in Table 1.

Figure 3: Model of the abdominal aorta and outlet nomenclature.

Figure 4: (a) Prescribed time history of aortic flow rate at the inlet and (b) schematic representation of boundary RCR block for open loop model of the abdominal aorta.

Figure 5: Flow rate reconstruction at abdominal aorta outlets using OLS and RVM regression. Dashed lines represent multiscale model flow rates, regressions are shown using continuous lines and shaded areas are used to highlight 95\% confidence regions.

Figure 6: Training residual distributions for flow rates in abdominal aortic model for various sampling ratios $m /\left(m_{t}-m_{w}\right)$. Outlets 9,10 and 12 (right and left exterior iliac artery and left inferior renal artery, respectively) are characterized by the largest regression errors. RVM: relevance vector machines, OLS: ordinary least squares.

Figure 7: Inlet and outlets for aortic arch model with coronary arteries.

Figure 8: Schematic representation of the simulated closed loop model used to investigate coronary artery flow.

Figure 9: Outlet flow rate reconstructions using OLS and RVM for coronary circulation model. 
Figure 10: Training residual distributions for flow rates in coronary model. Outlets 1 and 23 (outlet in the descending aorta and aortic inlet, respectively) are characterized by the largest regression errors.

Figure 11: Approximation of flow rate and pressure at the major outlets for the coronary circulation model. Multiscale model flow rates and pressures are compared with those obtained by manually tuned resistances and reconstructed using RVM.

Figure 12: Approximation of flow rates and pressures at the minor outlets for the coronary circulation model. While pressures are generally well captured at the coronary outlets, a tolerable but worse agreement is reached at low flow rates.

Figure 13: Ventricular PV-loops for the coronary circulation model. The graphs show how the RVM technique closely approximates the multiscale model PV-loop.

Figure 14: Agreement between outlet pressures and flow rates for the multiscale and proposed condensed RVM model of the abdominal aorta.

Figure 15: Outlet sensitivities for coronary model OLS and RVM surrogates. Darker colors indicate higher sensitivity to the pressure at a specific outlet; values on each row sum up to $100 \%$. Outlets whose pressure drives the flow rate at other outlets are identified as the main aortic outlet, Lca5 (due to presence of a coronary bypass graft) and Rca3 (main outlet of the right coronary artery).

Figure 16: Outlet sensitivities for OLS and RVM abdominal aorta surrogates. Darker colors indicate higher sensitivity to the pressure at a specific outlet; values on each row sum up to $100 \%$. The sensitivities computed using sparse regression highlight the importance of the aortic inflow (in open loop models where this quantity is prescribed) and the outlet on the right iliac artery. The SMA branch pressure affects renal and SMA flow rate.

Figure 17: Noise amplification factors of pressure/flow rate surrogates determined using OLS or RVM regressions. The noise amplification factors obtained thought RVM regression are significantly lower than those obtained from OLS. This is consistent with the fact that we were not able to determine working surrogates using OLS.

Table 1: Representative values of resistance and inertance characteristics for circulation in aorta and coronary arteries in healthy adults. Length of aorta is considered as the sum of average values of ascending, descending and thoracic aorta in healthy adults, discussed in Dotter et al. (1950). The quantities $\rho$ and $\nu$ denote blood density and viscosity, respectively, while $L$ and $d$ are the length and diameter of the selected physiologic system. 
Table 2: Characterization of boundary RCR circulation blocks at every interface $\Gamma_{i}$. 
Figure 1

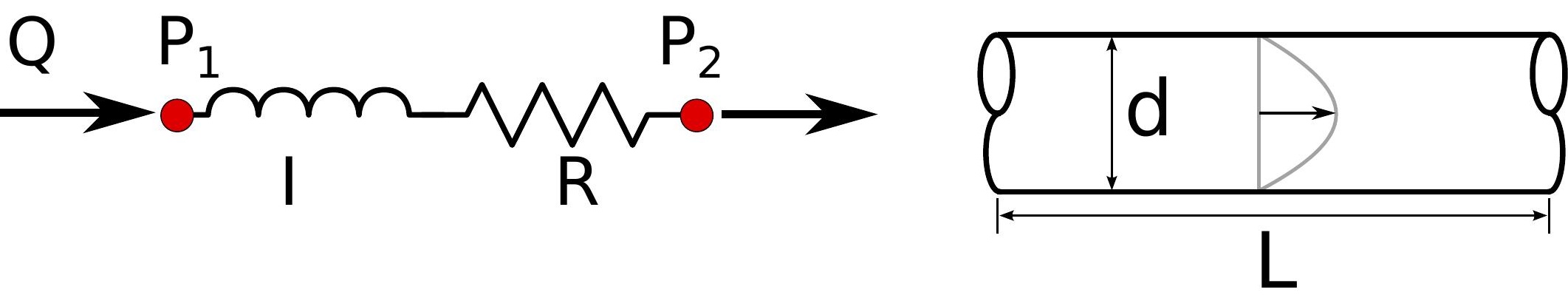



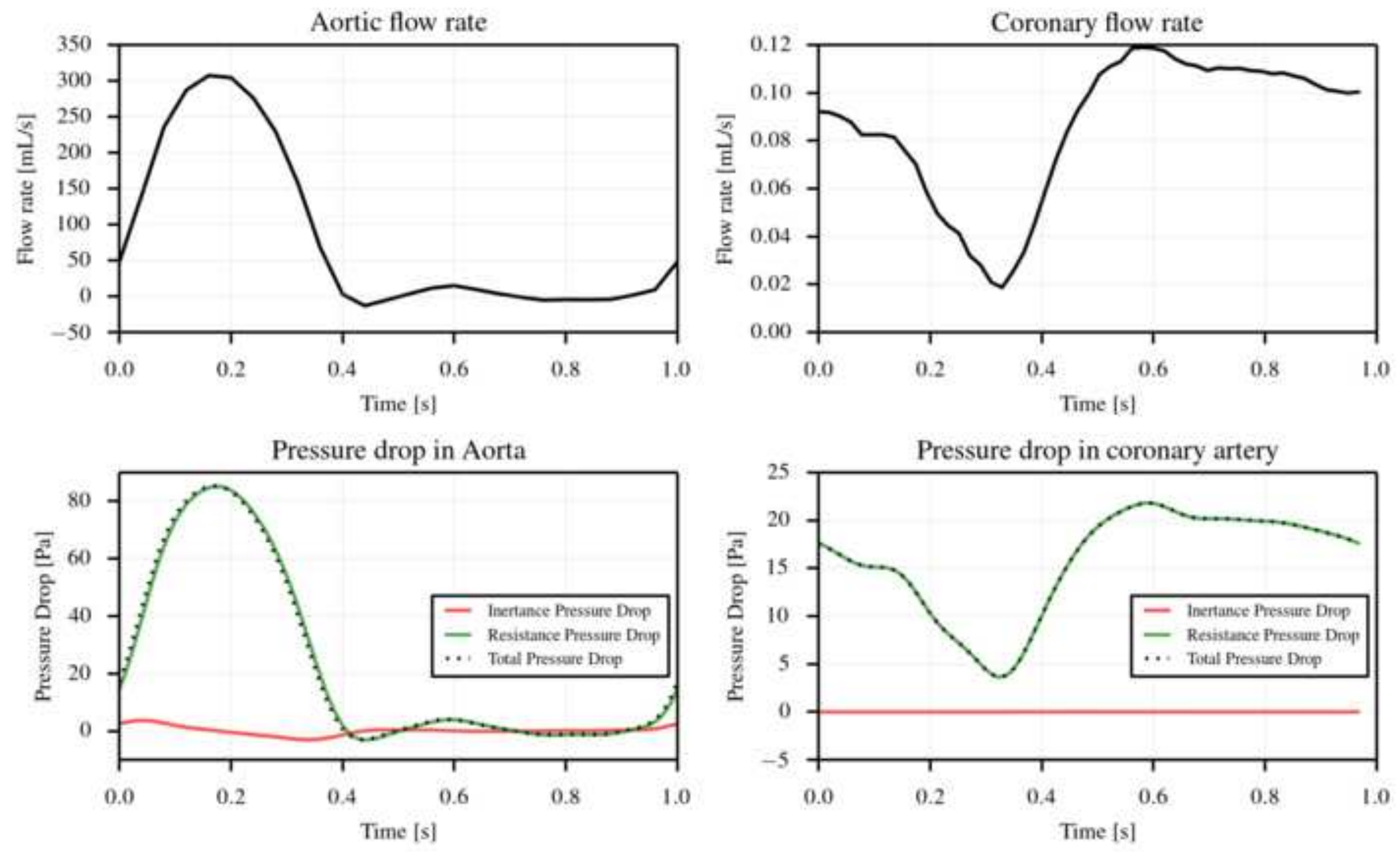


\section{Aortic inlet flow rate}

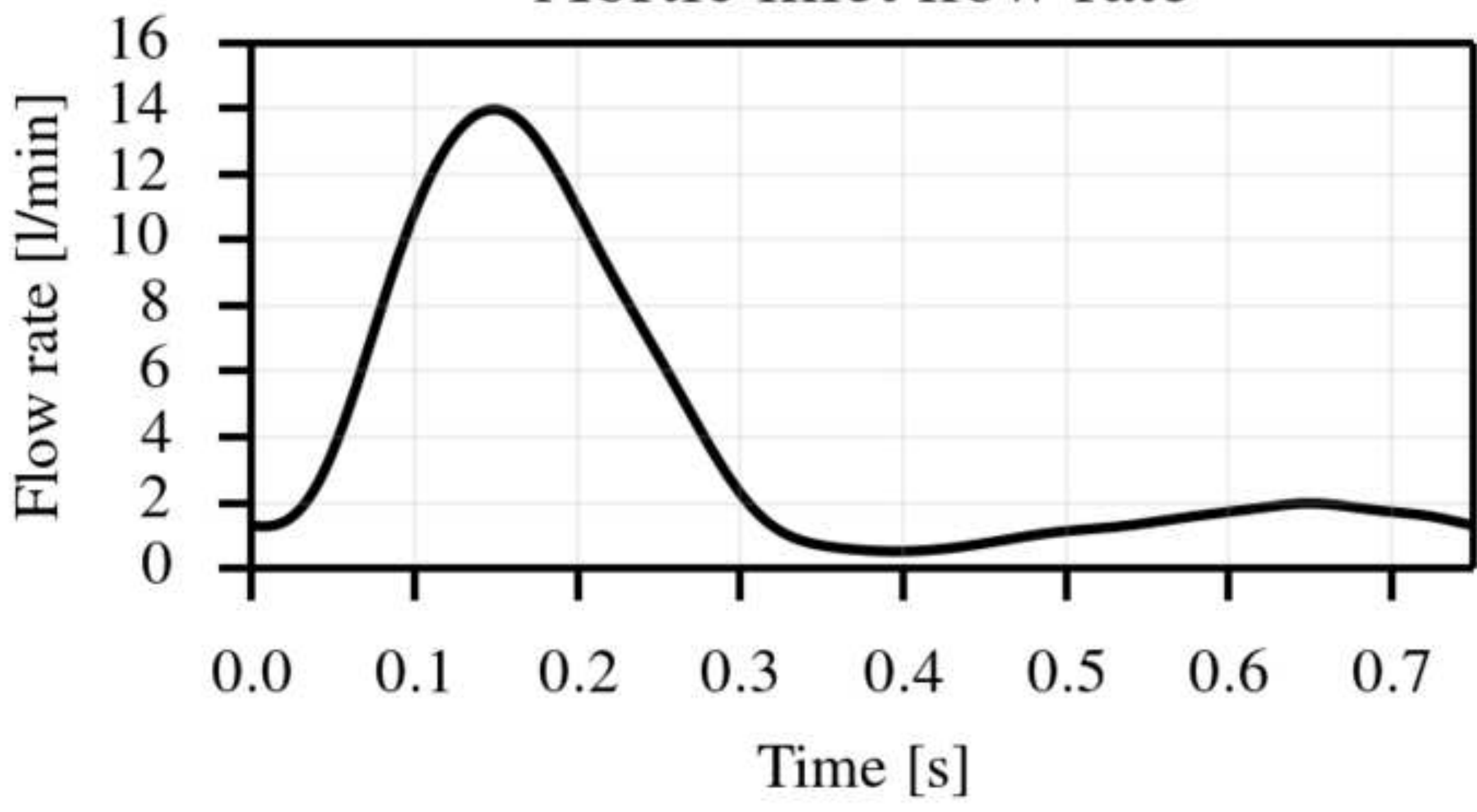




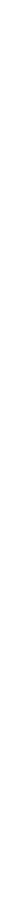

Figure $4 \mathbf{b}$ 

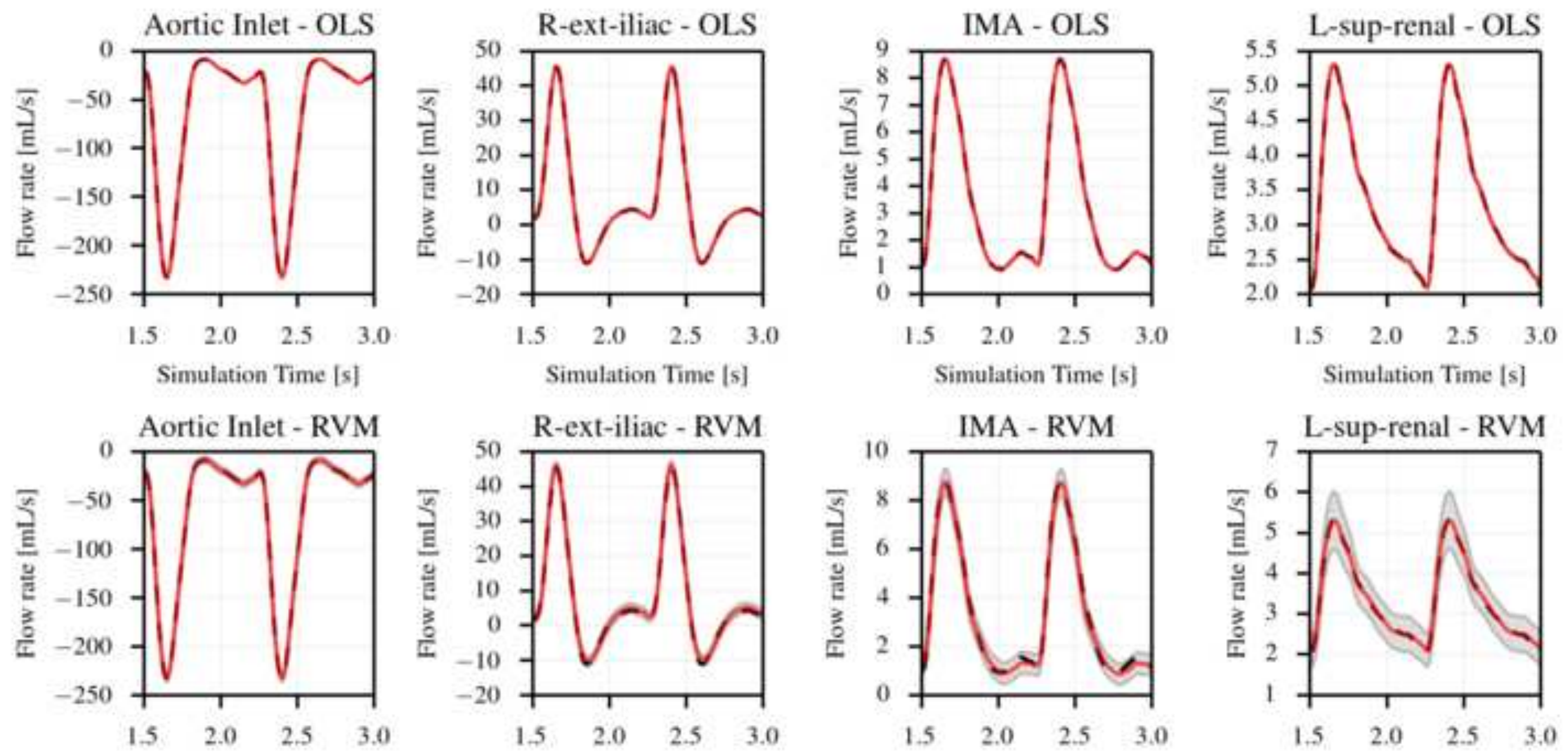

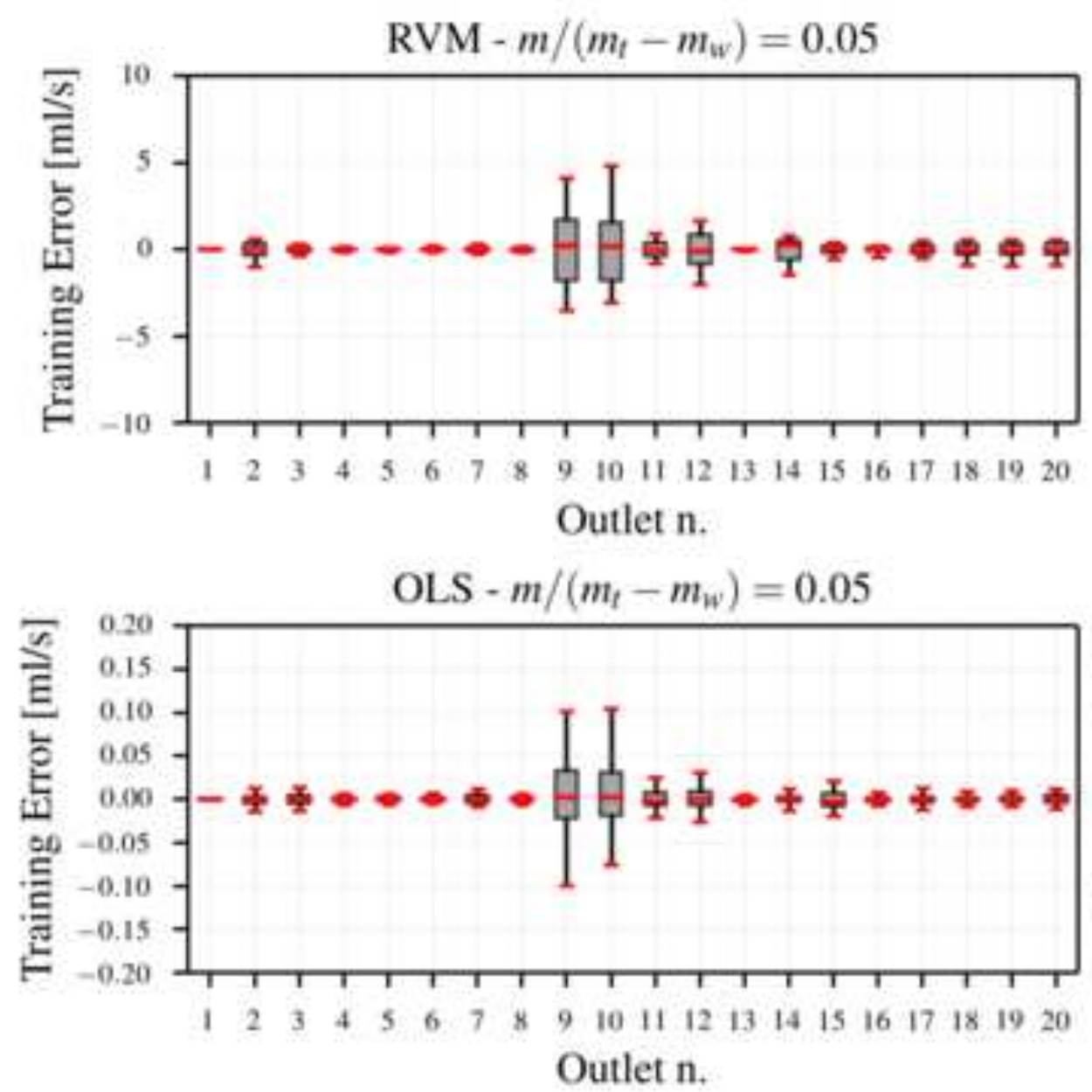
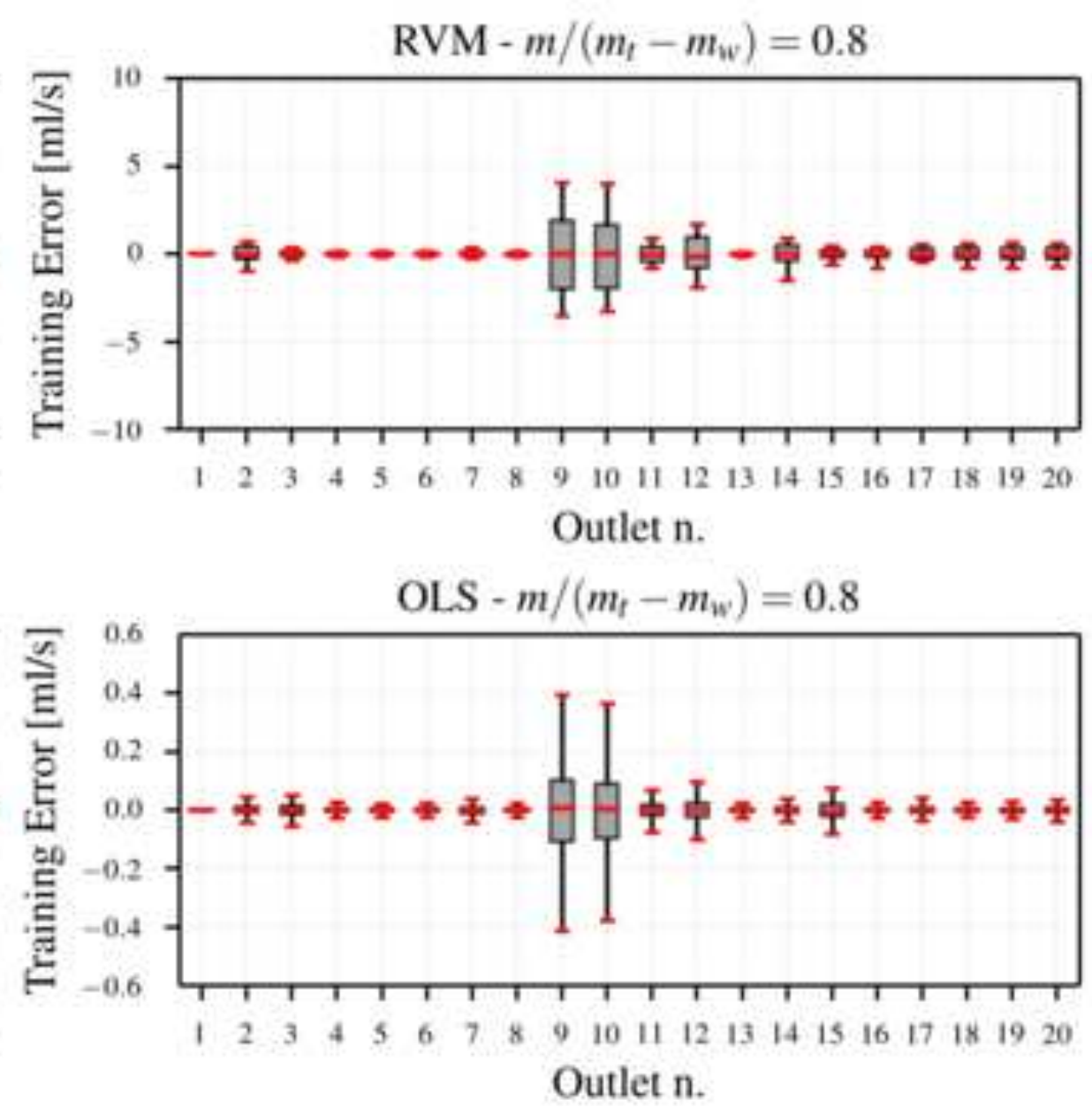


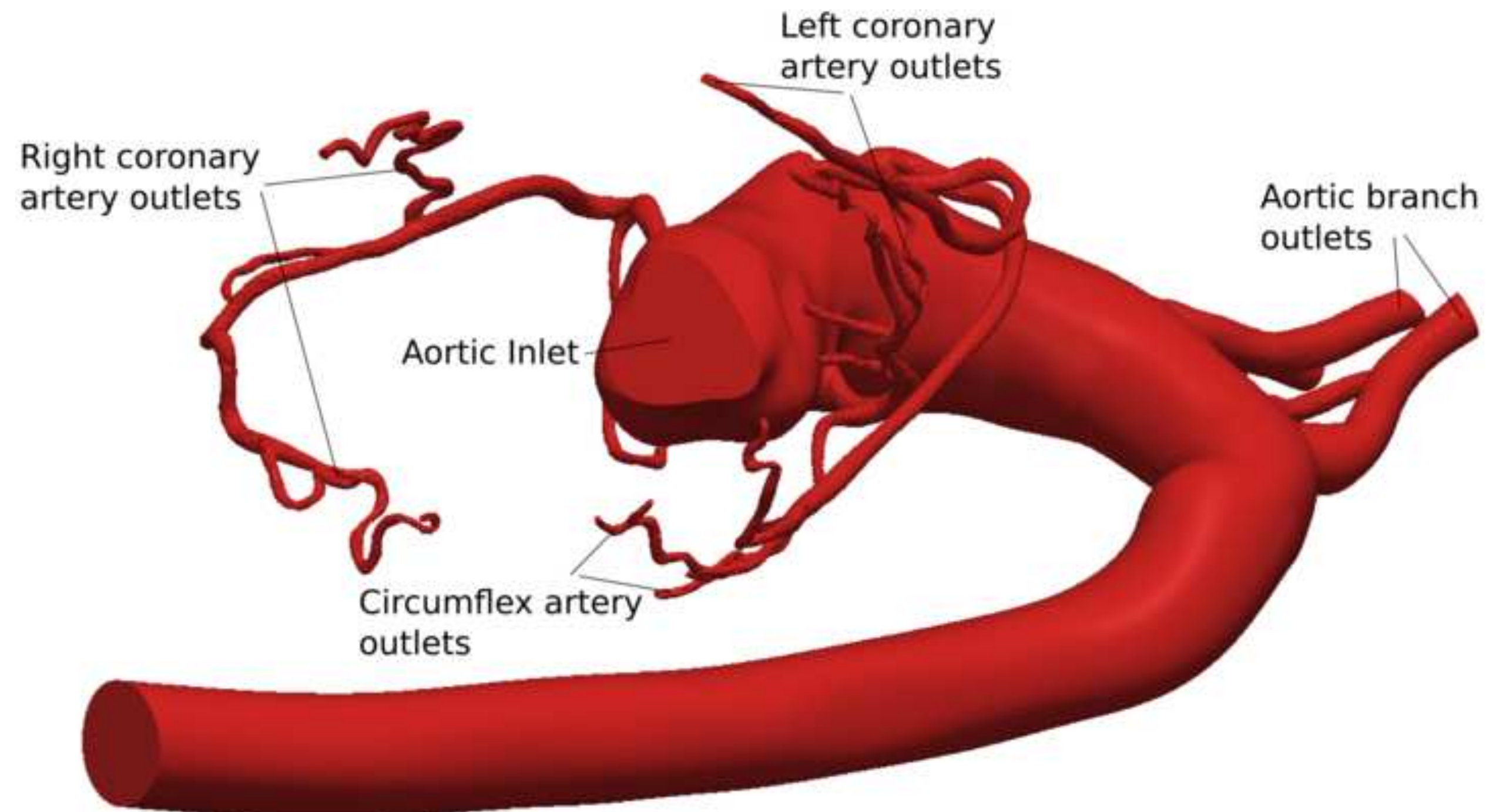




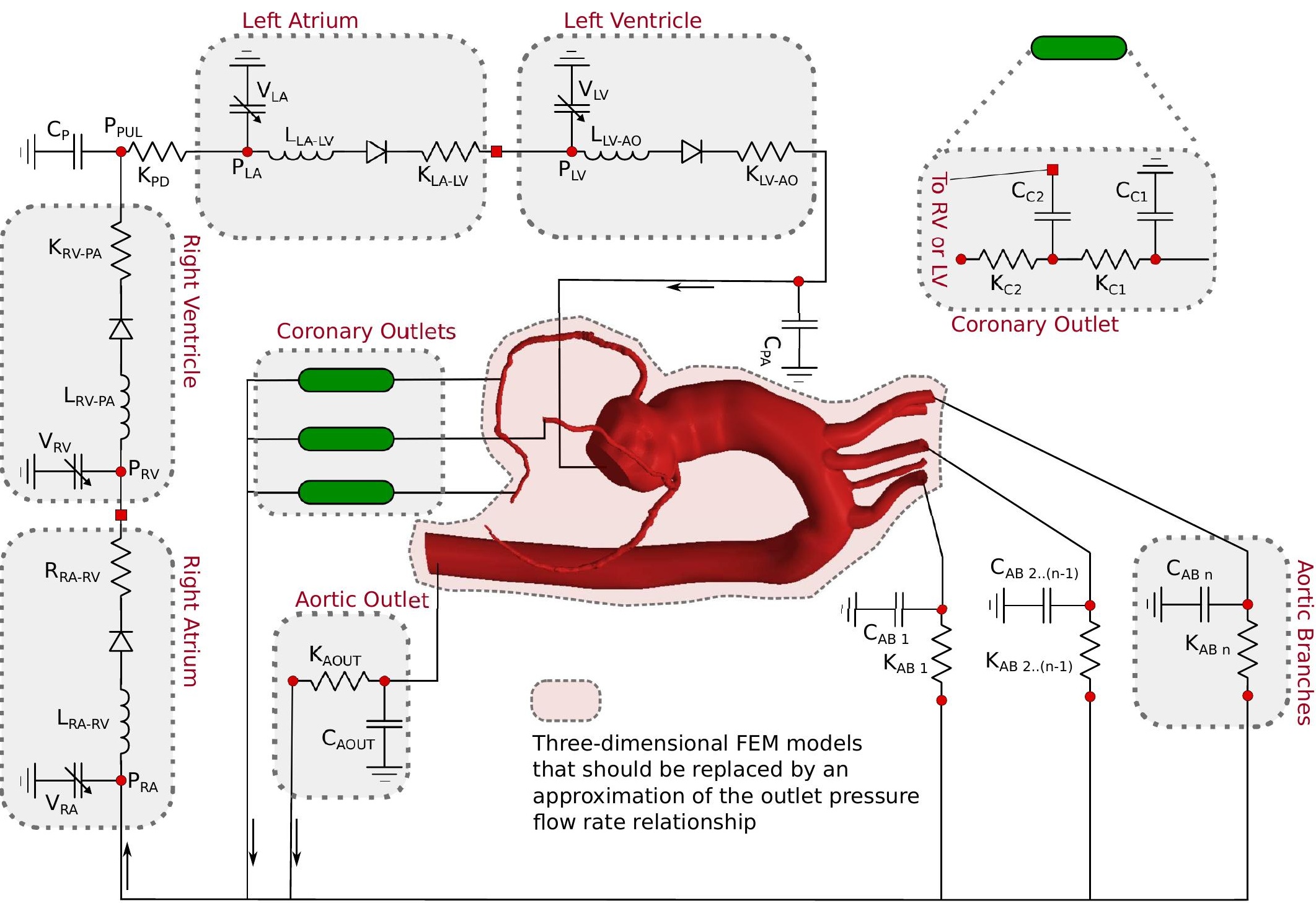




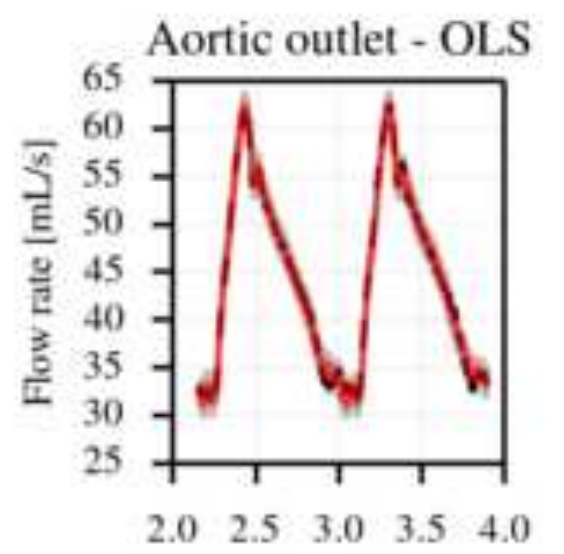

Simulation Time [s]

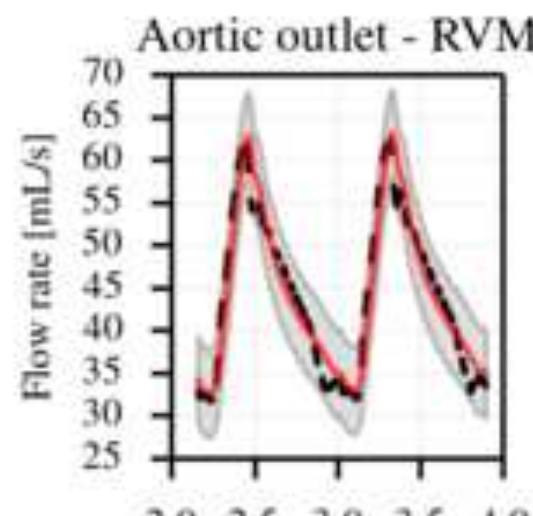

$\begin{array}{lllll}2.0 & 2.5 & 3.0 & 3.5 & 4.0\end{array}$
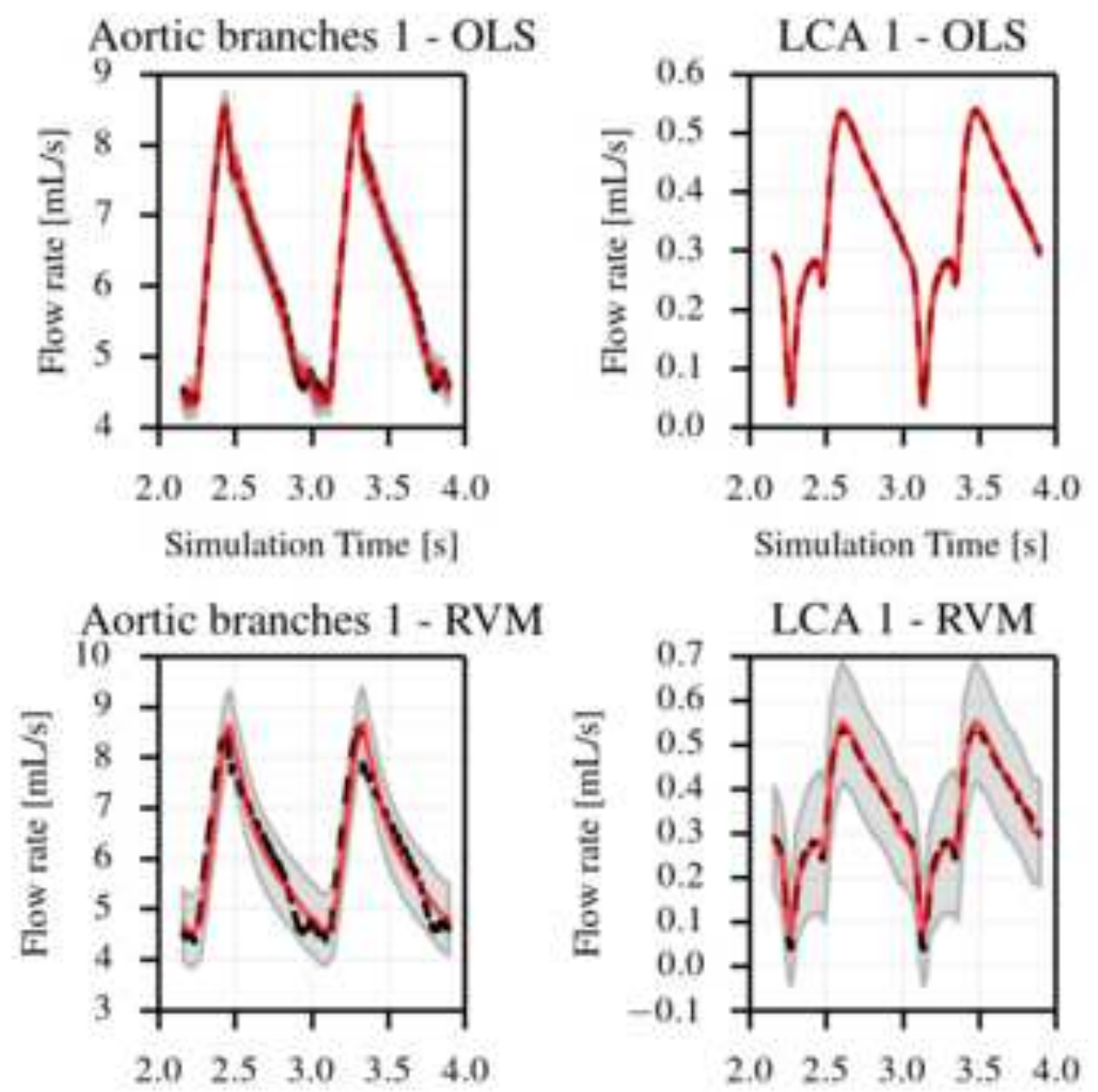

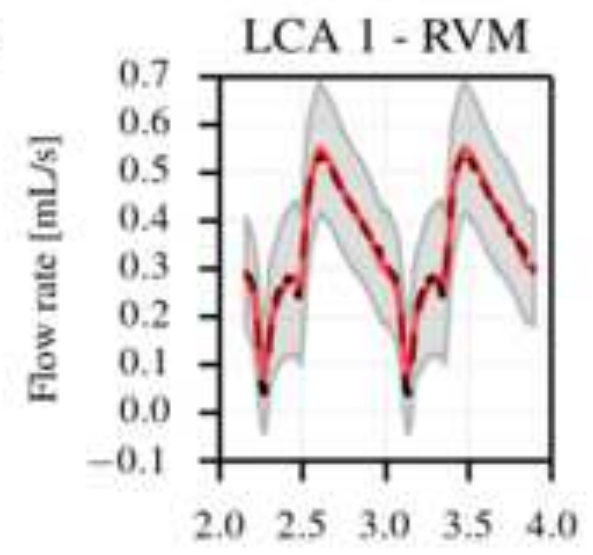

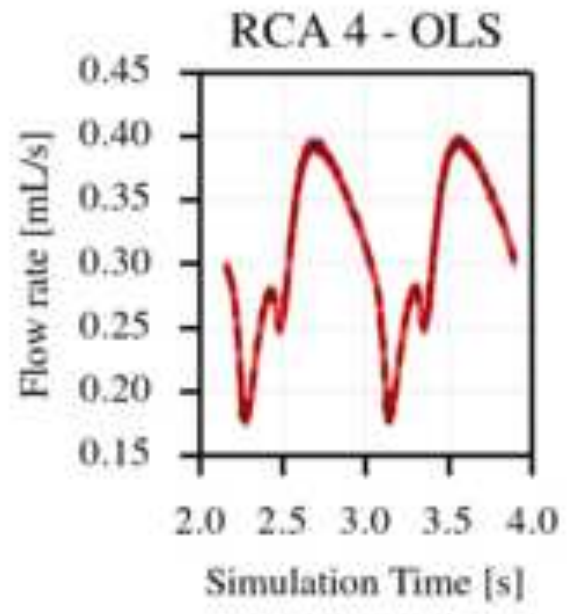

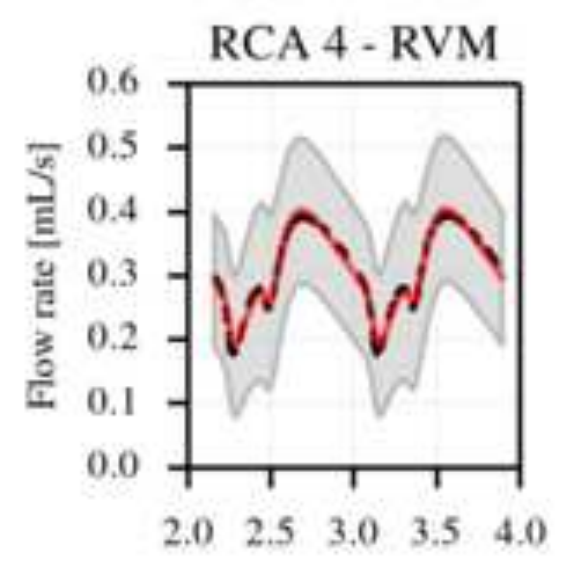



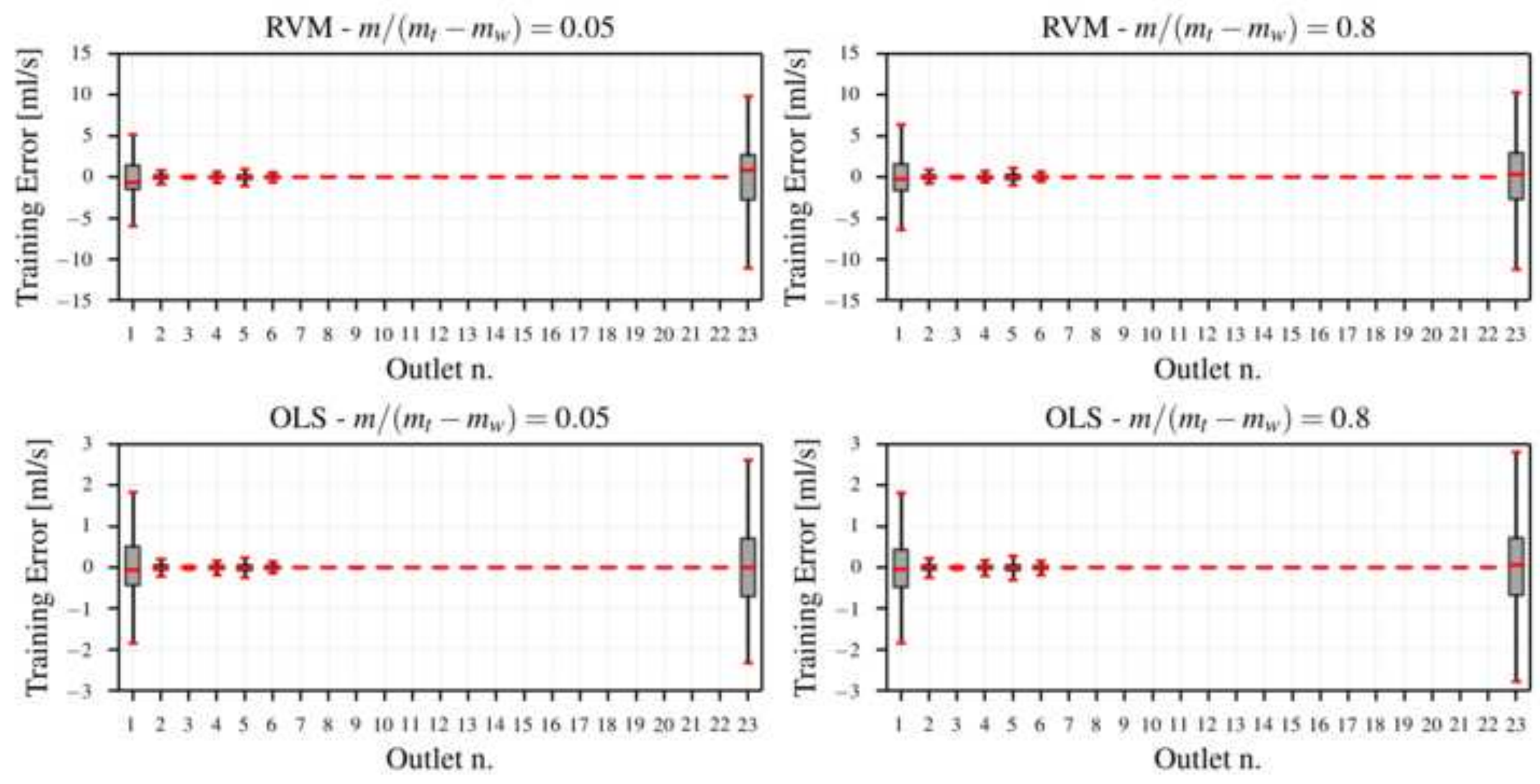

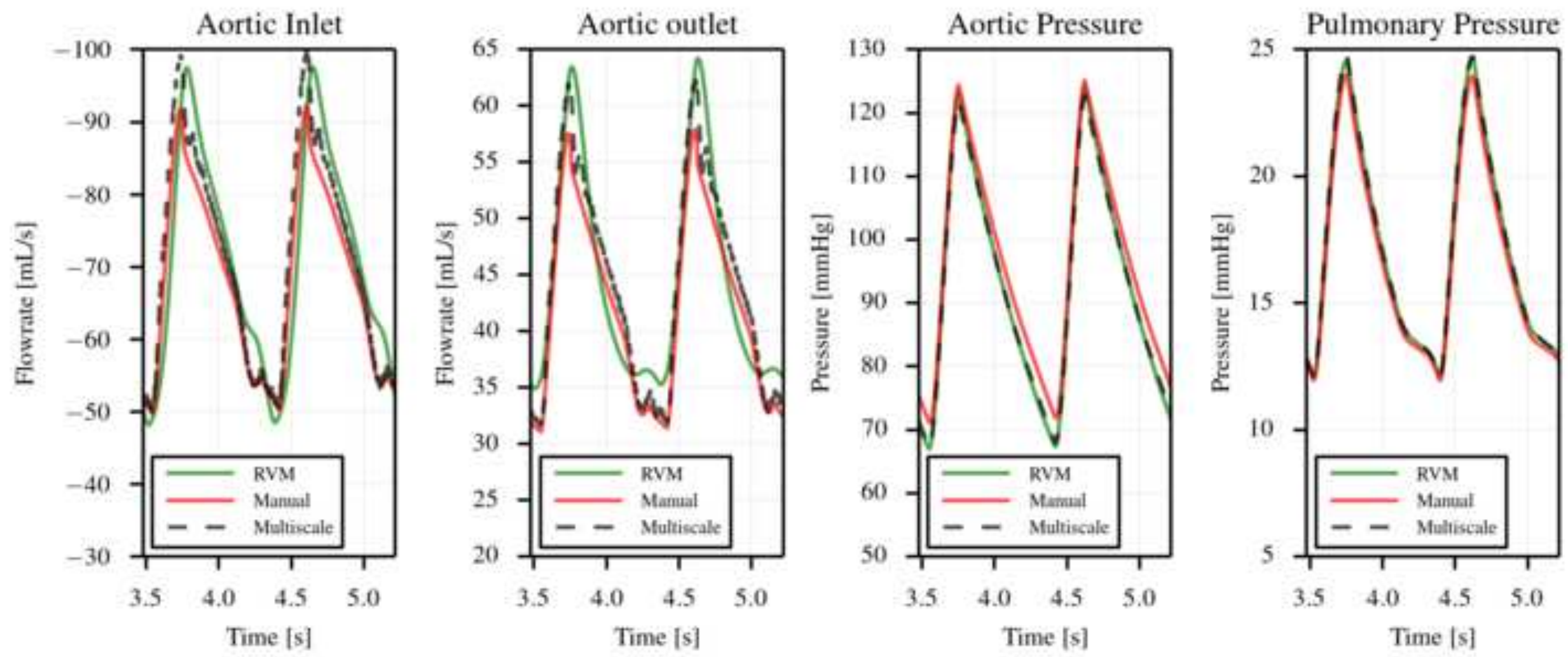

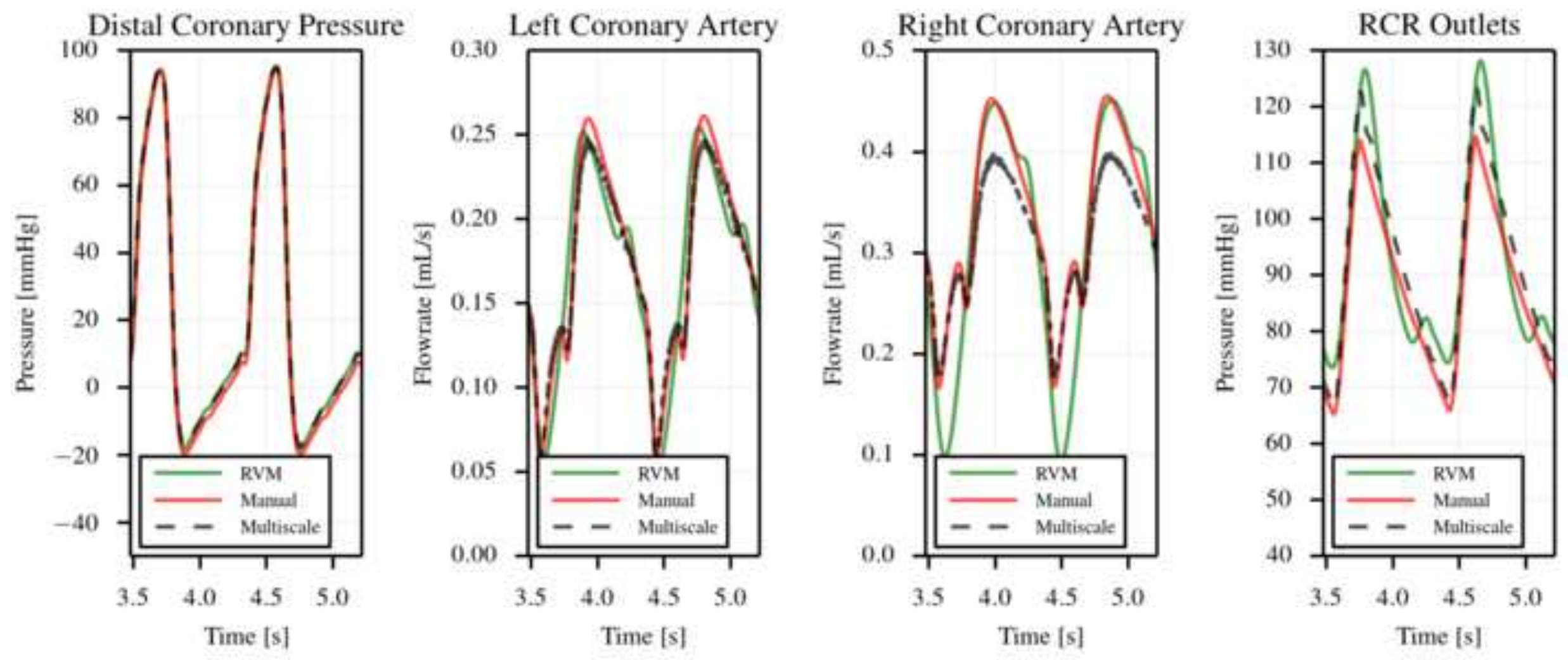

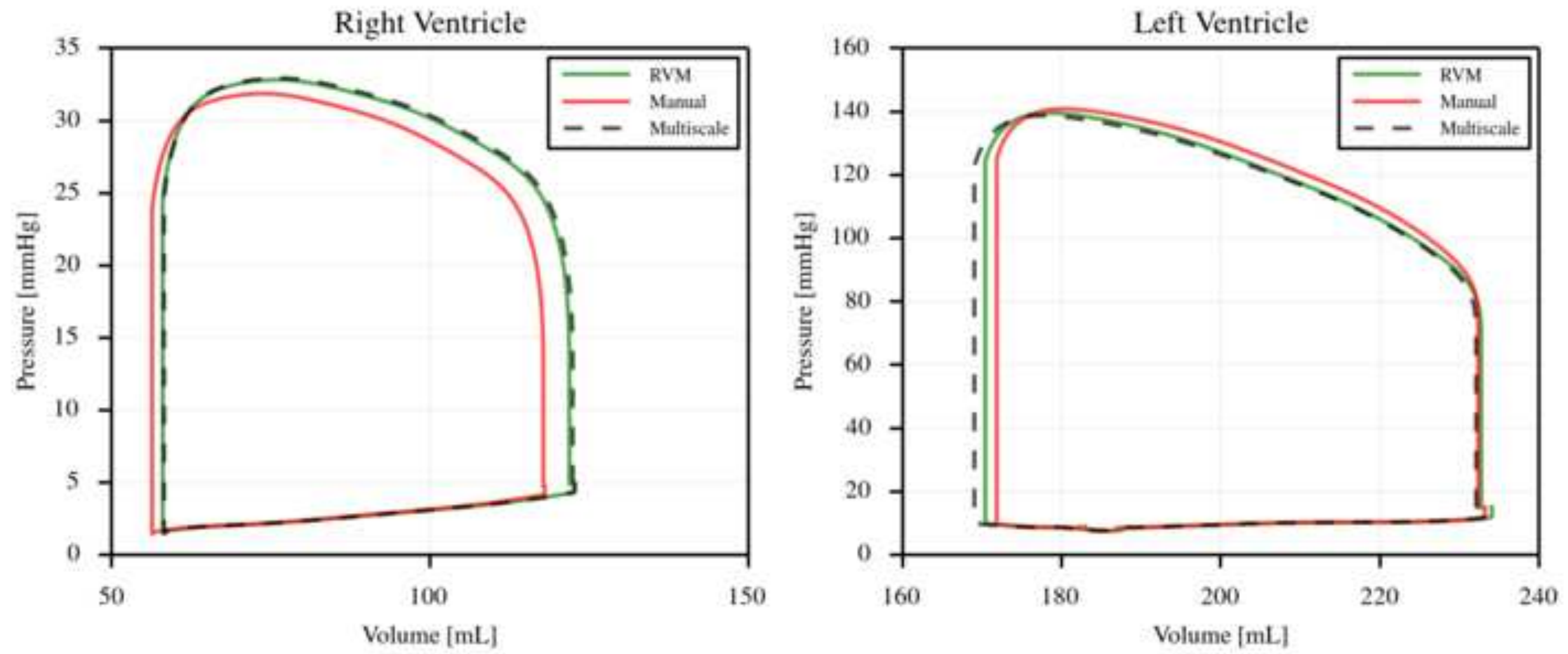

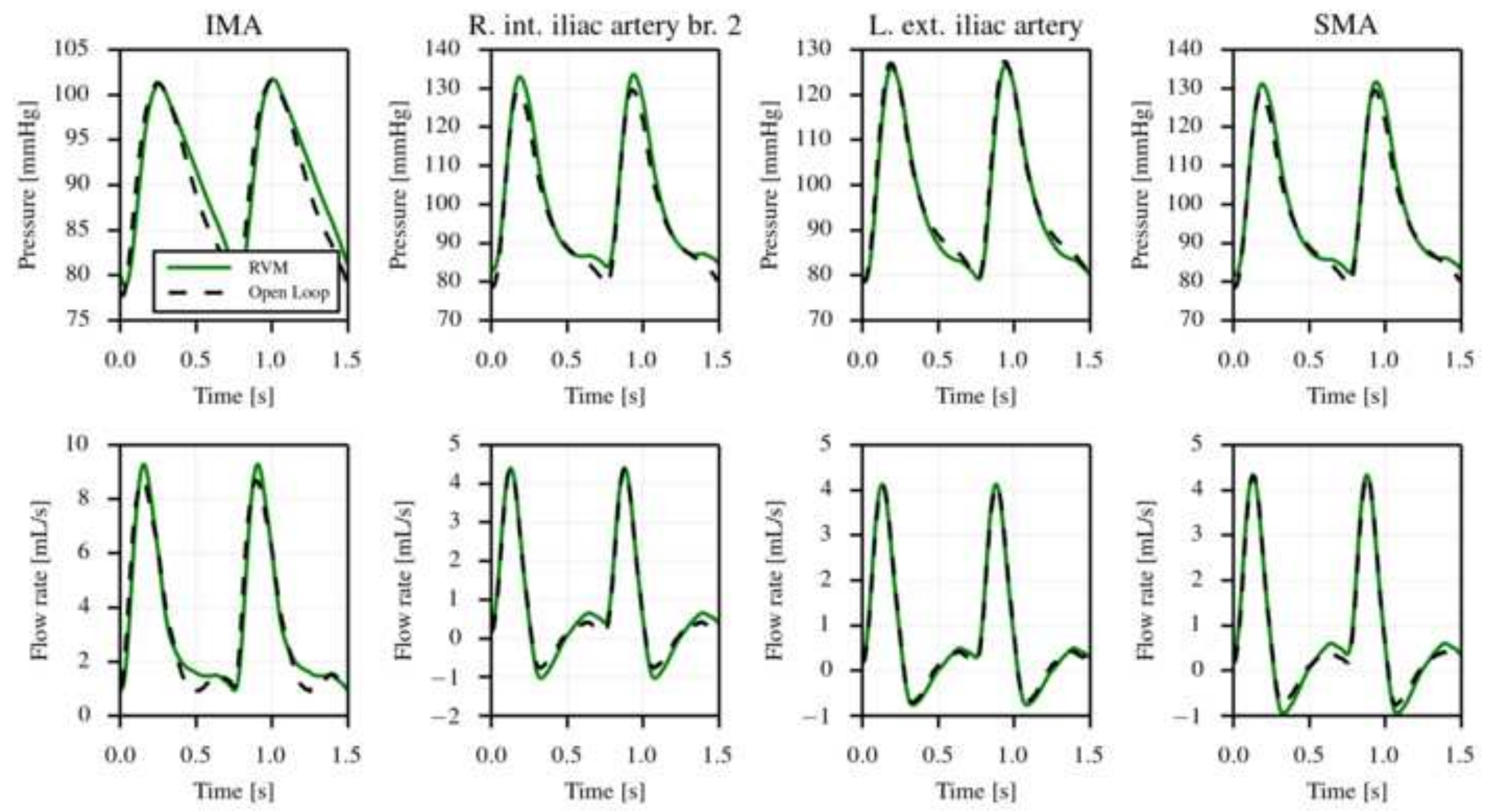

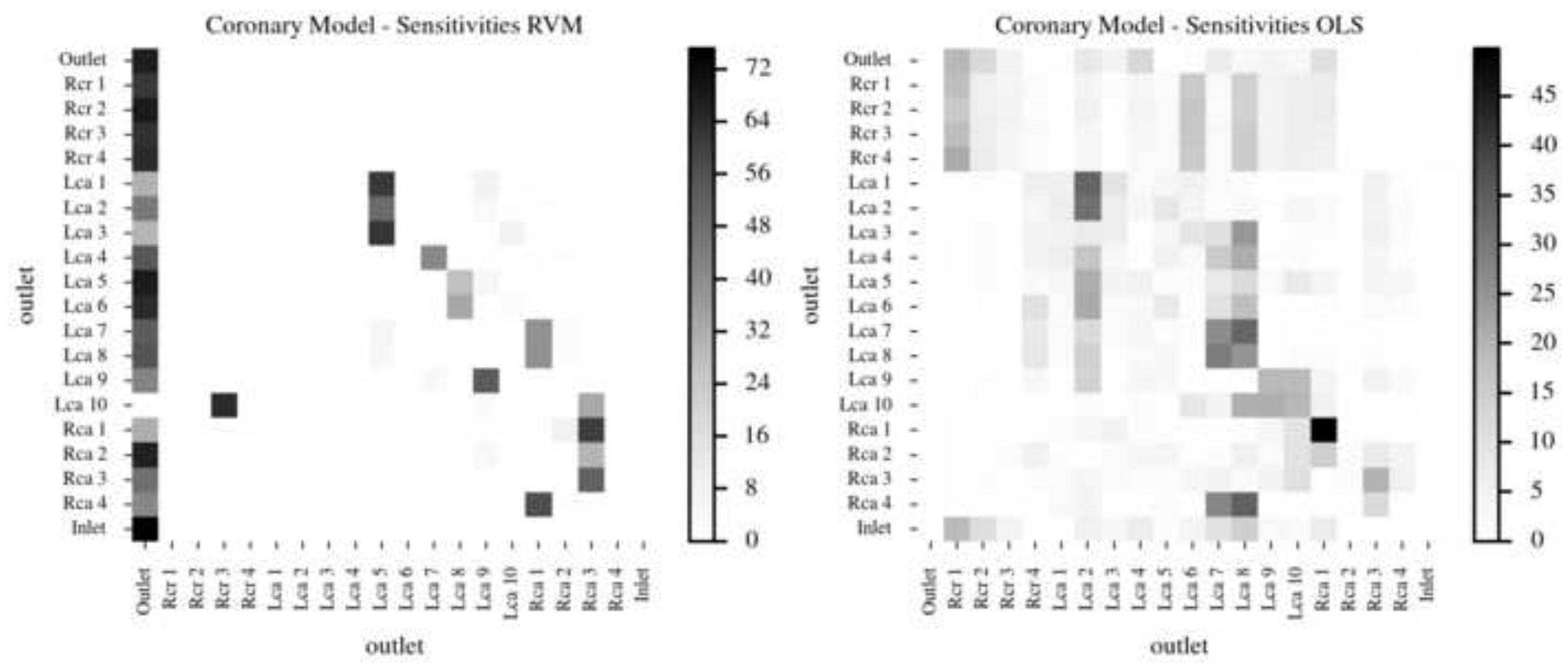

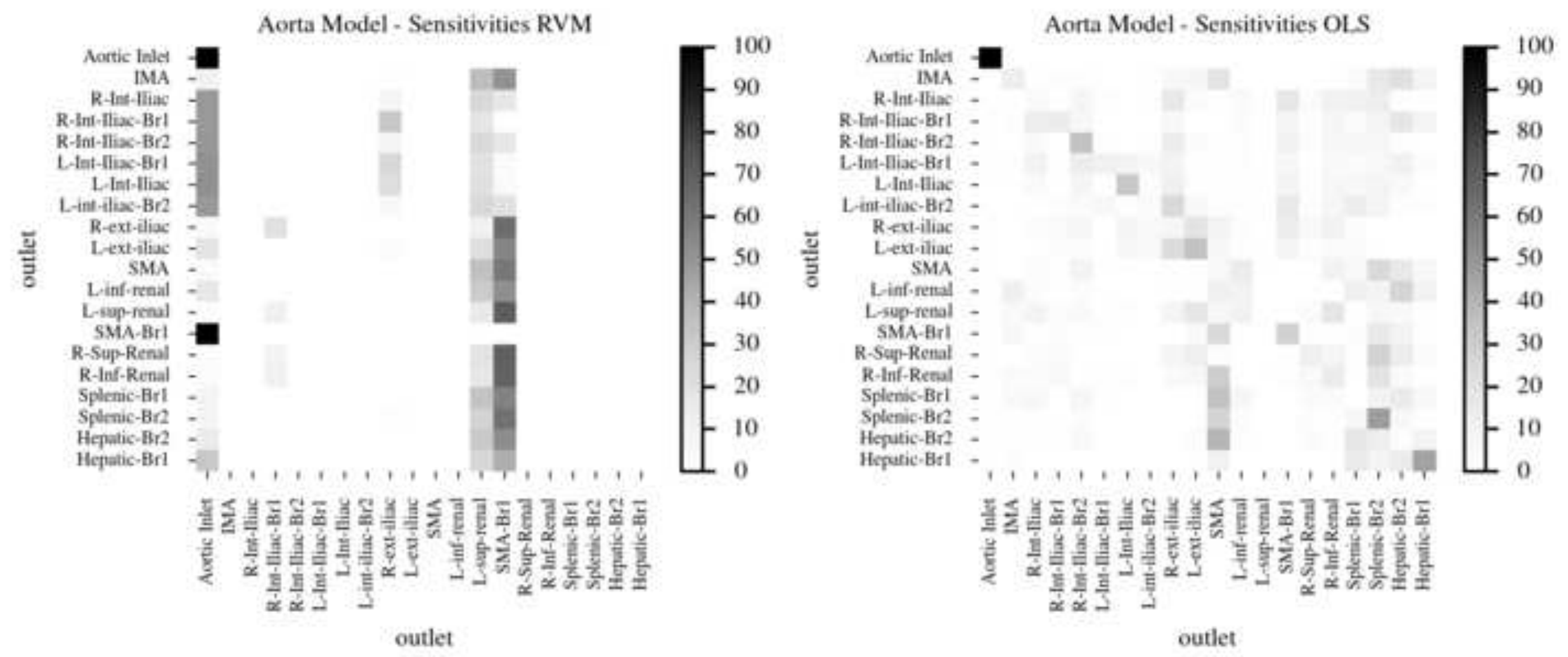

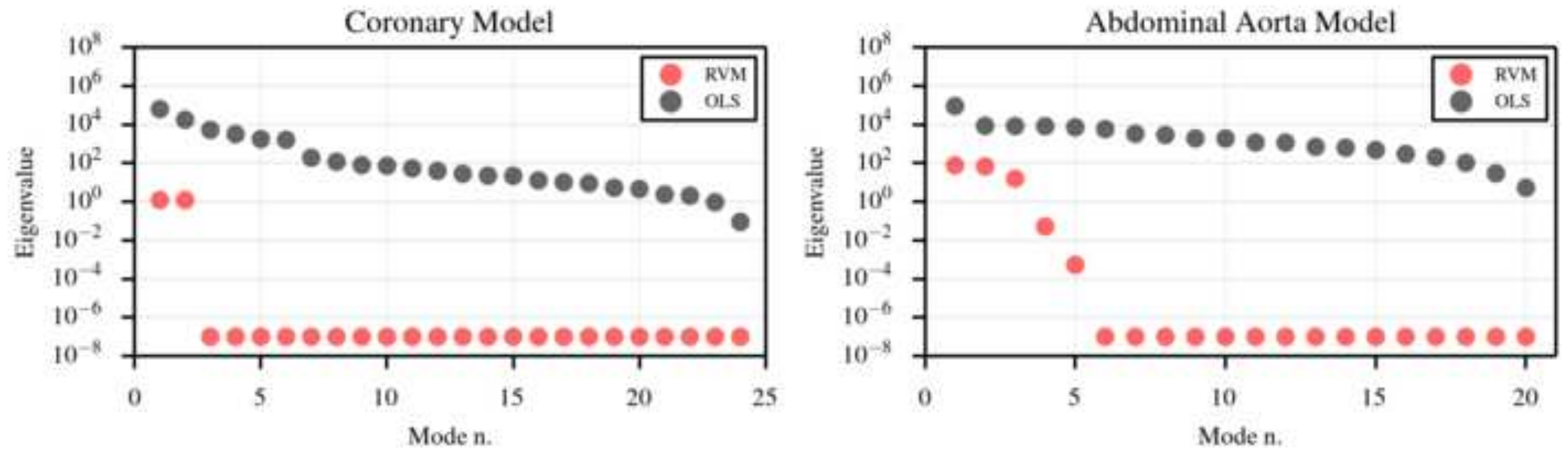